\title{
Multiphase buoyant plumes with soluble drops or bubbles
}

\author{
Shigan $\mathrm{Chu}^{*}$ \\ Department of Mechanical Engineering, Johns Hopkins University, Baltimore, Maryland 21218, USA \\ Andrea Prosperetti ${ }^{\dagger}{ }^{\dagger}$ \\ Department of Mechanical Engineering, University of Houston, Houston, Texas 77204, USA
}

(Received 6 September 2018; published 29 August 2019)

\begin{abstract}
This paper presents the results of a scaling study of bubble and drop plumes in a stratified ambient. Use is made of a standard integral model of the top-hat type, which can be reduced to one of the Gaussian type by a simple transformation. The focus of the work is on the effects of the dissolving material on the plume dynamics. It is pointed out that, for a drop plume, the loss of buoyancy due to dissolution can be compensated by a lightening of the ambient liquid associated with the dissolved drop material, or even aggravated if the density of the solution is greater than that of the undissolved drops. For bubbles, these effects are compounded by the volume expansion due to the falling hydrostatic pressure. This process is not important in deep water, where the peel height is smaller than the water depth, but can be significant in shallow water, where the two may be comparable. With a focus on the analysis of a point-source, three important parameters are identified. The first one compares the drop/bubble dissolution rate with the rise time to the neutral height (the level at which the plume density equals the ambient density), the second one accounts for the effect of the dissolved material on the liquid density, and the third one is the drop/bubble rise velocity compared with the characteristic plume velocity.
\end{abstract}

DOI: 10.1103/PhysRevFluids.4.084306

\section{INTRODUCTION}

Buoyant two-phase plumes in an ambient liquid are a frequent occurrence in nature (e.g., gas seepage from the ocean floor, hydrothermal vents), in environmental engineering (e.g., the oxygenation and destratification of water bodies), and in other situations; recent reviews are given in Refs. [1,2]. An occurrence that has garnered considerable attention has been the plume produced by the major oil spill caused by the Deepwater Horizon accident in the Gulf of Mexico in 2010 (see, e.g., Refs. [3-5]). In a plume of this type thermal buoyancy plays a relatively minor role, as the oil gushing out of the ground quickly mixes and reaches thermal equilibrium with the ambient water (see, e.g., Ref. [6]). Buoyancy is provided, rather, by the release of liquid hydrocarbons, less dense than water, and by the free hydrocarbon gas which forms ascending bubbles and possibly hydrate particles.

The present paper is a continuation of an earlier study [7] devoted to bubble plumes in a stratified ambient up to the first peel height. In that paper we neglected the dissolution of the bubbles and their volume expansion due to the falling pressure accompanying their rise. The first approximation is justified for sparingly soluble gases. The second one is appropriate for situations in which the peel

*schu13@jhu.edu

†Also at: Faculty of Science and Technology and J. M. Burgers Center for Fluid Dynamics, University of Twente, 7500AE Enschede, The Netherlands; aprosperetti@uh.edu 
height is a small fraction of the water depth so that the hydrostatic pressure changes are small. This point has been confirmed, e.g., by the results reported in Ref. [8] in which dissolution was found to be more important than volume change. Both assumptions are also relevant in very shallow water with very strong stratification, a situation often encountered in laboratory experiments.

In the present paper we allow for the effects of dissolution and variable hydrostatic pressure, and we consider plumes produced by drops in addition to bubbles. Unlike previous studies, we consider the effect that the dissolving drop/bubble material has on the density of the plume liquid, an aspect that has attracted scant attention to date. An exception is Ref. [9], which is the first study in which bubble shrinking due to both chemical reaction and dissolution were considered in a general form. In that study, bubbles were treated as passive scalars with a negligible slip velocity, an approximation that is not made in this paper.

In keeping with much of the previous theoretical work on the subject (see, e.g., Refs. [6,9-13]), we adopt an integral model as in our previous study. A RANS study was carried out in Ref. [11] and, more recently, two-fluid models and Lagrangian-Eulerian models based on Navier-Stokes equations have been developed (see, e.g., Refs. [11,14-17]). References [11,18] compared these models with the integral approach finding a good agreement on several fundamental aspects of the plume behavior. The results of Ref. [18] confirm this conclusion except in the flow development region very near the source.

There are several theoretical studies that include gas dissolution in bubble plumes. On the basis of an integral model and an entrainment hypothesis, Ref. [13] reports on the dissolution of an oxygen plume in a stratified lake. The authors also carry out a systematic study of source conditions such as initial bubble radius, oxygen volume rate and initial plume area. Their calculations are based on a measured stratification profile which limits somewhat the generality of their results. The RANS study of Ref. [11] considers dissolution of oxygen and nitrogen bubbles in a uniform, rather than stratified, ambient liquid. A carbon dioxide bubble plume was studied in Ref. [19] and compared with experimental results. The authors used a double plume model to predict the intrusion height finding results in general agreement with data, if with a large scatter. The results are reported in dimensional form which prevents their generalization to different situations.

In the present contribution we make an effort to identify the key dimensionless parameters that have the greatest influence on the dynamics of the system. For the deep-water case, we find that these parameters are essentially three. The first one compares the drop/bubble dissolution rate with the rise time to the neutral height (at which the plume density equals the ambient density), the second one accounts for the effect of the dissolved material on the liquid density, and the third one is the drop/bubble rise velocity compared with the characteristic plume velocity. In shallow water, the water depth compared with the neutral height must be added to the list.

Among other considerations, the extension of our previous model presented here is motivated by the observation, in connection with the Deepwater Horizon accident, that low-molecular-weight hydrocarbons, both liquid (benzene, toluene, ethylbenzene, xylenes, so called BTEX) and gaseous (methane, ethane, propane, butane) dissolved completely or partially as crude oil drops and natural gas bubbles rose in the deep ocean [4,5]. As a result, several carbon-rich intrusion layers were formed, with an observed persistence of months [20]. We find that the results for the peel and neutral heights are significantly affected by the dissolution rate and density modification of the plume liquid.

The multiphase plumes studied here bear some similarity with the single-phase thermal plumes encountered in one form of single-phase double-diffusive convection (see, e.g., Refs. [21-23]). In both cases salt provides the negative buoyancy, while upward momentum is provided by the liquid itself in one case, and by the ascending drops/bubbles dragging the liquid in the other. There are two further points of contact with conventional double-diffusion. First, much as the cold water entrained in a thermal plume gradually dilutes the source of buoyancy, the dissolution of bubbles or drops in a multiphase plume causes the buoyancy to decrease even though, as we show later, the soluble material dissolved in the water may decrease its density and restore, to some extent, the buoyancy lost by dissolution. Second, the multiple layers formed in single-phase double-diffusion 
are reminiscent of the multiple peel and intrusion heights formed in multiphase plumes when the plume source is sufficiently deep (see, e.g., Refs. [9,24]). A major difference between the two cases lies in the horizontal extension, which is typically relatively small in the multiphase case due to the localized nature of the drop/bubble source, while it is typically much larger in conventional double-diffusion as encountered, for example, in the ocean.

The formation of low-molecular-mass hydrates is possible at the temperature and pressure of the very deep ocean (see, e.g., Refs. [25,26]). We do not attempt to account for this process as its modeling is subject to considerable uncertainties $[8,25,27]$ and its inclusion would prevent us from obtaining general results.

\section{INTEGRAL MODEL FOR BUBBLE AND DROP PLUMES}

As in several other earlier studies (see, e.g., Refs. [13,24,28,29]), in this paper we consider monodisperse bubble or drop plumes. While the assumption of monodispersity is an approximation, its severity is mitigated by the fact that there is a significant size range (between 1 and $30 \mathrm{~mm}$ for bubbles and a comparable but smaller range for drops (see, e.g., Ref. [30]) in which the rise velocity of drops and bubbles depends rather weakly on their size. We also neglect the possibility of break-up and coalescence, the former because of the weakness of turbulence in many water bodies (except possibly in the top water layers), the latter because in natural waters the surface of bubbles and drops gets quickly contaminated so that coalescence is inhibited. It is also known that bubble coalescence is inhibited by dissolved salts (see, e.g., Refs. [31,32]). Several investigators have developed models to predict the bubble size distribution in an underwater blowout (recent references are Refs. [33,34]), but these models do not seem to have yet been incorporated into full plume models. We also neglect the possible presence of a horizontal current. The speed of these currents at depth is usually of the order of a few $\mathrm{cm} / \mathrm{s}$, and cannot therefore induce a horizontal drift of drops/bubbles nor have a significant effect on their vertical rise velocity. The plume axis may bend, but the horizontal drag is large enough that the drops/bubbles will be transported by the current so that, in a Lagrangian sense, they will not be affected by it. A horizontal current may however affect the liquid entrainment into the plume (see, e.g., Ref. [35]), which, without horizontal current, occurs at a rate of approximately $10 \%$ of the plume velocity. This may be the most significant effect of a weak horizontal current, which therefore becomes non-negligible when its velocity becomes about $10 \%$ of the plume rise velocity.

We begin by presenting the equations appropriate for a drop plume because, as will be explained in next section, the same equations apply for bubble plumes in deep water. After this we consider the case of bubble plumes in shallow water.

The theoretical framework of the current study is essentially the same as in our previous paper [7], with the exception that it incorporates drop/bubble dissolution and the consequent liquid density modification as described in Sec. IV.

\section{A. Drop plume}

We consider explicitly single-component drops. The multicomponent case can be treated analogously provided the interaction between the different components is properly accounted for as shown in Ref. [36]. The case in which only one component of the drop is soluble, while the remaining ones are insoluble, is briefly sketched in the Appendix. Some of the relations that we derive here are applicable also to the case of bubble plumes, which justifies the use of the words drop/bubble in this case.

The procedure by which the equations for the integral model are derived is well known. We demonstrate it here for the volume fraction of the disperse phase referring the reader to the literature (see, e.g., Ref. [37]) and to our earlier paper [7] for details on the derivation of the complete model.

In steady conditions the balance equation for the disperse phase is

$$
\nabla \cdot\left[\alpha_{d} \rho_{d}\left(\mathbf{w}+\mathbf{w}_{d}\right)\right]=\pi d^{2} n_{d} h_{d}\left(\rho_{d, \mathrm{dslv}}-\rho_{\mathrm{sat}}\right) .
$$


In the left-hand side, $\alpha_{d}=\frac{\pi}{6} d^{3} n_{d}$, with $n_{d}$ the number density of drops/bubbles with diameter $d$ and density $\rho_{d}$, is the volume fraction of the disperse phase, assumed monodisperse, $\mathbf{w}$ is the liquid velocity, and $\mathbf{w}_{d}$ the drop/bubble drift velocity relative to the liquid. In the right-hand side, $h_{d}$ is the mass transfer coefficient, $\rho_{d \text {,dslv }}$ is the density of the drop/bubble material dissolved in the liquid, and $\rho_{\text {sat }}$ is the saturation value. We integrate this equation over a thin horizontal "slice" of the plume with thickness $d z$ and apply the divergence theorem to find

$$
\frac{d}{d z} \int \alpha_{d} \rho_{d}\left(w+w_{d}\right) d A=\int \pi n_{d} d D \operatorname{Sh}\left(\rho_{d, \mathrm{dslv}}-\rho_{\mathrm{sat}}\right) d A,
$$

where $w=w(z)$ and $w_{d}=w_{d}(z)$ are the vertical velocities (positive upward); the integration is over the area $A=A(z)$ of the plume cross section, and $\mathrm{Sh}=h_{d} d / D$ is the Sherwood number. The small ring-shaped surface at the edge of the plume does not give any contribution because, by the very definition of the volume of the plume, there is no transport of the disperse phase through its edge.

The analogous integral form for the balance of the dissolved drop/bubble material is

$$
\frac{d}{d z} \int\left(1-\alpha_{d}\right) \rho_{d, \mathrm{dslv}} w d A=-\int \pi n_{d} d D \operatorname{Sh}\left(\rho_{d, \mathrm{dslv}}-\rho_{\mathrm{sat}}\right) d A .
$$

The appearance of the same quantity with opposite signs in the right-hand side of these two equations guarantees the conservation of the soluble material.

The integral form of the balance equation for the dissolved salt is

$$
\frac{d}{d z} \int\left(1-\alpha_{d}\right)\left(\rho_{s}-\rho_{s, a}\right) w d A+\frac{d \rho_{s, a}}{d z} \int\left(1-\alpha_{d}\right) w d A=0 .
$$

Here $\rho_{s}$ is the density of the dissolved salt in the plume and $\rho_{s, a}$ the ambient value of this quantity outside the plume. The second term accounts for the entrainment of ambient liquid into the plume, a process that does not affect the balance relations for the dissolved material since $\rho_{d \text {,dslv }}$ vanishes outside the plume.

The statement of conservation of the liquid mass flow rate in the plume is

$$
\frac{d}{d z} \int_{A}\left(1-\alpha_{d}\right) \rho w d A=2 \pi \rho_{a} \alpha_{e} w .
$$

Here $\alpha_{e}$ is the entrainment coefficient at the plume's edge which will be treated as a constant, as is appropriate for fully developed turbulent conditions; a discussion of this assumption can be found in Refs. [38,39]. The proportionality relation between the plume vertical velocity and the rate of entrainment, originally introduced in Ref. [40], can be justified on the basis of dimensional considerations noting the irrelevance of viscosity at high Reynolds numbers in fully developed turbulent conditions. On the same basis, the numerical value of $\alpha_{e}$ can be expected to be independent of the physical properties of the liquid but, because of this hypothesis, the considerations that follow are not directly applicable to laminar plumes. Frequently used values in the literature are $\alpha_{e}=0.083-0.085$ [28,38,41]. For a Gaussian profile of the flow fields, the values encountered in the literature are between 0.07 and 0.11 [42]. This range is supported by the recent DNS simulations of single-phase plumes in Ref. [16] where, however, a dependence on distance from the source was found. Such a dependence was also found in the recent LES numerical simulations of bubble plumes in Ref. [17], where the mean value over the entire height of the simulation was reported to be 0.086 and over the fully developed region 0.067 . A likely explanation for this dependence is the presence of a flow establishment zone (see, e.g., Ref. [18]). Since we are interested here in the basic aspects of buoyant plumes rather than precise numerical values, we feel justified in assuming a constant value for $\alpha_{e}$. We do not need to commit ourselves to a specific value because $\alpha_{e}$ will be absorbed in the dimensionless groups introduced later. It may be noted that conversion of the values of $\alpha_{e}$ reported for a Gaussian distribution to the case of the top-hat profile assumed in this 
work requires multiplication by $\sqrt{2}$ so that, for example, $\alpha_{e}=0.083$ for a Gaussian profile becomes $\alpha_{e}=0.083 \sqrt{2}=0.117$ for a top-hat profile.

With the neglect of the viscous and turbulent stresses at the edge of the plume, the total momentum flow rate in the plume is

$$
\frac{d}{d z} \int\left[\left(1-\alpha_{d}\right) \rho w^{2}+\alpha_{d} \rho_{d}\left(w+w_{d}\right)^{2}\right] d A=\int\left[\alpha_{d}\left(\rho_{a}-\rho_{d}\right)+\left(1-\alpha_{d}\right)\left(\rho_{a}-\rho\right)\right] g d A .
$$

The appearance of $\rho_{a}$ in the first term of the right-hand side is justified by noting that, if $\rho=\rho_{d}$, then the total buoyancy must be the area integral of $\left(\rho_{a}-\rho\right) g=\left(\rho_{a}-\rho_{d}\right) g$. It is argued in the Appendix that $\alpha_{d}$ can be neglected everywhere except in Eq. (2).

For simplicity, and to carry out a scaling analysis, we assume that all fields are uniform over the cross section $A$ of the plume, the so-called "top hat" approximation. However, as in other models (see, e.g., Refs. $[18,37,43]$ ), we allow for the disperse phase to occupy only a fraction of the plume cross sectional area. Thus, for the mass flux $m_{d}$ carried by the drops/bubbles, we let

$$
m_{d}(z) \equiv \int \alpha_{d} \rho_{d}\left(w+w_{d}\right) d A \simeq \lambda A \alpha_{d} \rho_{d}\left(w+w_{d}\right),
$$

in which $\lambda A$ is the area of the plume cross section occupied by drops or bubbles. For bubble plumes, $\lambda$ has been variously reported as 0.6 and 0.8 (cited in Ref. [12]). The calculations of Ref. [12] show negligible differences between $\lambda=0.8$ and 0.9 . The comparison between a detailed three-dimensional model and the integral model of Ref. [15] is reasonable assuming $\lambda=0.8$. No similar information seems to be available for drop plumes but, due to the smaller buoyancy of drops, turbulent transport may be expected to be effective, which would lead to an even smaller difference between the two cross sections for drops than for bubbles. In any event, it will be seen below that, in the limit of small disperse-phase volume fraction that we consider, the plume momentum is mostly affected by the total buoyancy exerted by the disperse-phase mass flux rather than by its spatial distribution over the plume cross section, so that the parameter parameter $\lambda$ has no influence on the model predictions.

While the flow rate of the drop/bubble material in the plume changes due to dissolution, the total flow rate of the number of drops/bubbles does not, at least as long as they do not completely dissolve. Of course, in principle, break-up and coalescence could also change the number of drops/bubbles but, as noted before, usually the turbulence environment in which plumes are encountered is not so violent as to cause these phenomena on a large scale. Hence, we feel justified in neglecting them. With these hypotheses, the rate of injection of drops/bubbles at the source,

$$
\Omega=\lambda n_{d}\left(w+w_{d}\right) A,
$$

remains a constant. Upon making a top-hat profile assumption in the right-hand side of Eq. (2), we may write

$$
\frac{d m_{d}}{d z}=-\frac{d m_{d, \mathrm{dslv}}}{d z} \simeq \frac{\pi \Omega d D \mathrm{Sh}}{w+w_{d}}\left(\rho_{d, \mathrm{dslv}}-\rho_{\mathrm{sat}}\right)
$$

independent of $\lambda$. It may be noted that, because of the constancy of $\Omega$, this equation can be reduced to

$$
\left(w+w_{d}\right) \frac{d}{d z}\left(\frac{\pi}{6} d^{3} \rho_{d}\right)=\frac{d}{d t}\left(\frac{\pi}{6} d^{3} \rho_{d}\right)=\pi d D \operatorname{Sh}\left(\rho_{d, \mathrm{dslv}}-\rho_{\mathrm{sat}}\right),
$$

where $d / d t$ denotes the Lagrangian derivative, which describes the dissolution of an individual drop/bubble.

Conservation of the soluble material requires that

$$
m_{d}+m_{d, \mathrm{dslv}}=m_{d 0},
$$


in which $m_{d 0}$ is the total mass flow rate of the soluble material injected by the source and

$$
m_{d, \mathrm{dslv}}(z) \equiv \int\left(1-\alpha_{d}\right) \rho_{d, \mathrm{dslv}} w d A \simeq \lambda_{d} A \rho_{d, \mathrm{dslv}} w
$$

in which $\lambda_{d}$ is the fraction of the plume cross-section containing an appreciable amount of dissolved material. Even in the absence of true turbulence, mixing in the plume is favored by the so-called pseudoturbulence, namely, the disordered motion caused by the rising disperse phase. Hence, we expect that $\lambda<\lambda_{d}$.

With

$$
m(z) \equiv \int_{A}\left(1-\alpha_{d}\right) \rho w d A \simeq A \rho w,
$$

the balance equation for the liquid mass flow rate in the plume becomes

$$
\frac{d m}{d z}=2 \pi b(z) \rho_{a} \alpha_{e} w=2 \alpha_{e} \sqrt{\pi \rho M},
$$

in which $b=\sqrt{A / \pi}$ is the radius of the plume and

$$
M \equiv \int\left[\left(1-\alpha_{d}\right) \rho w^{2}+\alpha_{d} \rho_{d}\left(w+w_{d}\right)^{2}\right] d A \simeq A \rho w^{2} .
$$

The ratio of the two terms in the integrand, representing the momentum flow rate of the disperse and continuous phases, is

$$
\frac{\alpha_{d} \rho_{d}\left(w+w_{d}\right)^{2}}{\left(1-\alpha_{d}\right) \rho w^{2}}=\frac{\alpha_{d}}{1-\alpha_{d}} \frac{\rho_{d}}{\rho}\left(1+\frac{w_{d}}{w}\right)^{2},
$$

and is therefore negligible for small $\alpha_{d}$ given that the other two factors are of order 1 or smaller. In effecting this estimate we have taken $\lambda=1$, which has no adverse consequences given that the conclusion of the argument is that $\alpha_{d}$ is negligible.

Turning now to the right-hand side of the momentum Eq. (6), we note that

$$
\alpha_{d}\left(\rho_{a}-\rho_{d}\right)+\left(1-\alpha_{d}\right)\left(\rho_{a}-\rho\right) \simeq \alpha_{d}\left(\rho_{a}-\rho_{d}\right)-\left(\rho-\rho_{a}\right),
$$

because $\alpha_{d} \ll 1$ (see Appendix), and

$$
\left|\frac{\rho_{a}-\rho}{\rho_{a}-\rho_{d}}\right| \ll 1
$$

given that the density difference due to the different salt content between the plume and the ambient water is much less than the density difference between water and organic liquids and, even more, gases. The difference between the densities of the plume water $\rho$ and of the ambient water outside the plume $\rho_{a}$ is due to the higher salt content of the former and to the presence of dissolved material. Since the concentration of both quantities is small, their contributions to the density superpose linearly and we may write

$$
\rho-\rho_{a}=\beta_{d} \rho_{d, \mathrm{dslv}}+\beta_{s}\left(\rho_{s}-\rho_{s a}\right)
$$

in which $\rho_{s}$ and $\rho_{s a}$ are salt densities inside and outside the plume, respectively, and

$$
\beta_{d}=\left.\frac{\partial \rho}{\partial \rho_{d, \mathrm{dslv}}}\right|_{\rho_{d, \mathrm{dslv}}=0}, \quad \beta_{s}=\left.\left.\frac{\partial \rho}{\partial \rho_{s}}\right|_{\rho_{s}=\rho_{s a}} \simeq \frac{\partial \rho}{\partial \rho_{s}}\right|_{\rho_{s}=0} .
$$

After substitution of (19), the right-hand side of Eq. (17) contains the three major contributions to the buoyancy of the plume, each one of which is associated to a corresponding flux, namely, the buoyancy flux due to the disperse phase

$$
F_{d}=\int \alpha_{d}\left(\rho_{a}-\rho_{d}\right)\left(w+w_{d}\right) g d A \simeq \lambda A \alpha_{d}\left(\rho_{a}-\rho_{d}\right)\left(w+w_{d}\right) g=\frac{\rho_{a}-\rho_{d}}{\rho_{d}} m_{d} g,
$$


the buoyancy flux due to salinity

$$
F_{s}=-\int\left(1-\alpha_{d}\right) \beta_{s}\left(\rho_{s}-\rho_{s, a}\right) w d A \simeq-A \beta_{s}\left(\rho_{s}-\rho_{s, a}\right) w
$$

and that due to the dissolved material

$$
\begin{aligned}
F_{d, \mathrm{dslv}} & =-\int\left(1-\alpha_{d}\right) \beta_{d} \rho_{d, \mathrm{dslv}} g w d A \simeq-\int \beta_{d} \rho_{d, \mathrm{dslv}} g w d A \\
& \simeq-\lambda_{d} A \beta_{d} \rho_{d, \mathrm{dslv}} g w=-\beta_{d} g m_{d, \mathrm{dslv}}
\end{aligned}
$$

Thus, upon expressing $F_{d}$ in terms of $m_{d}$ and $F_{d \text {,dslv }}$ in terms of $m_{d \text {,dslv }}$, the momentum balance relation Eq. (6) may be written as

$$
\frac{d M}{d z}=\frac{\rho_{a}-\rho_{d}}{\rho_{d}} \frac{m_{d} g}{w+w_{d}}-\beta_{d} g \frac{m_{d, \mathrm{dslv}}}{w}+\frac{F_{s}}{w} .
$$

Since $F_{s}$ is negative and continually decreasing, a level is reached where the right-hand side of this equation vanishes; we refer to this level as the neutral buoyancy height or, more simply, the neutral height. The right-hand side of Eq. (24) displays a structure analogous to that encountered in double-diffusive phenomena already mentioned in Sec. I.

Due to the accumulated inertia, the plume rises beyond the neutral height until its momentum vanishes at the peel height. At this level the density of the liquid in the plume is so much greater than that of the ambient liquid that the drops/bubbles are unable to lift it further. The plume liquid slumps back down spreading horizontally somewhat above the neutral height, as the further dilution due to the entrained liquid between the neutral height and the peel height has moved the equal-density level upward, as we have noted in out earlier paper [7]. Several papers can be found in the literature having as their objective the description of the "double-plume" structure, with the rising liquid in the inner plume and the descending liquid in the outer one (see, e.g., Refs. [6,44]), although this subject remains in need of further clarification.

From the balance Eq. (4) for the dissolved salt we derive an equation for the salinity flux, namely,

$$
\frac{d F_{s}}{d z}=-m N^{2}
$$

in which

$$
N^{2}=-\frac{g}{\rho_{a 0}} \frac{d \rho}{d z}
$$

is the Brunt-Väisäla frequency in terms of the ambient density at the source $\rho_{a 0}$. In view of the small effect that the difference in salt content has on all terms except buoyancy, in keeping with the Boussinesq approximation, we replace $\rho$ and $\rho_{a}$ by $\rho_{a 0}$ everywhere except in Eq. (22) of the buoyancy flux due to salinity $F_{s}$. Given the strong decrease in the concentration of the disperse phase that occurs already near the source, the density of dissolved material $\rho_{d \text {,dslv }}$ will be much less than the saturation density. We neglect this term in the following, writing Eq. (9) as

$$
\frac{d m_{d}}{d z} \simeq-\frac{\pi \Omega d D S h}{w+w_{d}} \rho_{\text {sat }}
$$

Furthermore, from Eqs. (7) and (8), we see that

$$
d=\left(\frac{6 m_{d}}{\pi \Omega \rho_{d}}\right)^{1 / 3}
$$

which can be used to express $d$ in Eq. (27). 


\section{NONDIMENSIONALIZATION}

At distances from the source of the order of the neutral height $L_{n}$, the mass of fluid entrained in the plume is much larger than the mass emitted by the source so that the mass balance Eq. (14) gives, in order of magnitude,

$$
\frac{\pi b^{2} \rho w}{L_{n}} \sim 2 \pi b \rho_{a} \alpha_{e} w .
$$

In view of the smallness of the difference between the plume liquid density $\rho$ and the ambient density $\rho_{a}$, from this relation we deduce that the plume radius at the neutral height is of the order of

$$
b\left(z=L_{n}\right) \sim 2 \alpha_{e} L_{n} .
$$

The dominant terms in the right-hand side of the momentum equation are those due to the positive buoyancy of the disperse phase and to the negative buoyancy of the entrained salty water, with the buoyancy due to the dissolved material playing a relatively minor role. Disregarding the dissolution of the disperse phase, so that $F_{d} \simeq F_{d 0}$, at the neutral height the salinity and drop/bubble buoyancy fluxes are in approximate balance so that

$$
F_{s} \simeq-\frac{w}{w+w_{d}} F_{d 0}=-\frac{w}{w+w_{d}} \frac{\rho_{a 0}-\rho_{d 0}}{\rho_{d 0}} m_{d 0} g,
$$

where, as before, the subscript 0 refers to values at the source. Since, at the source, no entrainment has yet taken place, so that $F_{s}=0$, and no material has yet dissolved, so that $F_{d \text {,dslv }}=0$, the total buoyancy flux reduces to the disperse-phase contribution $F_{d 0}$. At the height $L_{n}$ above the source the plume radius is of the order of $2 \alpha_{e} L_{n}$, as just noted, and therefore, upon using Eq. (31) in the salinity flux Eq. (25), we may write

$$
-\frac{F_{s}}{L_{n}} \sim \frac{1}{1+w_{d} / w} \frac{F_{d 0}}{L_{n}} \sim \pi\left(2 \alpha_{e} L_{n}\right)^{2} w N^{2} \sim \pi\left(2 \alpha_{e} L_{n}\right)^{2} L_{n} N^{3} .
$$

Since, as is well-known, $N^{-1}$ is of the order of the rise time of the plume liquid from the source to the neutral height [45], which implies that $w \sim L_{n} N$, and since $w / w_{d}$ does not differ much from unity, we find

$$
L_{n}=\left(\frac{F_{d 0}}{4 \pi \alpha_{e}^{2} \rho_{a 0} N^{3}}\right)^{1 / 4} .
$$

The equal sign defines the quantity $L_{n}$ which the previous argument has shown to be of the order of the neutral height. Since $L_{n}$ equals the neutral height $h_{n}$ only in order of magnitude, we retain two different symbols for these two quantities; a precise determination of $h_{n}$ will be given below. This result agrees in order of magnitude with that in the classical work of Ref. [46] and, in its detailed form, with that found in our previous paper [7].

The previous arguments suggest the use of the length scale $L_{n}$ and of the time scale $N^{-1}$ to nondimensionalize the plume-related quantities:

$$
\begin{gathered}
z^{*}=\frac{z}{L_{n}}, \quad m^{*}=\frac{m}{\pi\left(2 \alpha_{e} L_{n}\right)^{2} \rho_{a 0}\left(N L_{n}\right)}, \\
M^{*}=\frac{M}{\pi\left(2 \alpha_{e} L_{n}\right)^{2} \rho_{a 0}\left(N L_{n}\right)^{2}}, \quad F_{s}^{*}=\frac{F_{s}}{\pi\left(2 \alpha_{e} L_{n}\right)^{2} \rho_{a 0}\left(N L_{n}\right)\left(N^{2} L_{n}\right)},
\end{gathered}
$$

in which $N L_{n}$ and $N^{2} L_{n}$ are of the order of the plume velocity and acceleration. As already noted, for simplicity, and in the spirit of the Boussinesq approximation, we systematically replace the liquid densities $\rho$ and $\rho_{a}$ by $\rho_{a 0}$ everywhere except in the salinity buoyancy term. 
We use the values at the source to nondimensionalize the disperse-phase quantities:

$$
d^{*}=\frac{d}{d_{0}}, \quad m_{d}^{*}=\frac{m_{d}}{m_{d 0}}=\rho_{d}^{*}\left(d^{*}\right)^{3}, \quad \rho_{\mathrm{sat}}^{*}=\frac{\rho_{\mathrm{sat}}}{\rho_{\mathrm{sat}, 0}} .
$$

Here $\rho_{\text {sat }, 0}$ is the drop/bubble material solubility at the source and $\rho_{d}^{*}=\rho_{d} / \rho_{d 0}$.

With these definitions, the previous Eqs. (14), (24), (25), and (27) become

$$
\begin{gathered}
\frac{d m^{*}}{d z^{*}}=\sqrt{M^{*}} \\
\frac{d M^{*}}{d z^{*}}=\frac{1-\left(\rho_{d 0} / \rho_{a 0}\right) \rho_{d}^{*}}{1-\rho_{d 0} / \rho_{a 0}} \frac{m_{d}^{*} / \rho_{d}^{*}}{w^{*}+V_{N}}+\Lambda \frac{1-m_{d}^{*}}{w^{*}}+\frac{F_{s}^{*}}{w^{*}}, \\
\frac{d F_{s}^{*}}{d z^{*}}=-m^{*}, \\
\frac{d m_{d}^{*}}{d z^{*}}=-\Theta \frac{\mathrm{Sh}^{*}}{w^{*}+V_{N}}\left(\frac{m_{d}^{*}}{\rho_{d}^{*}}\right)^{1 / 3} \rho_{\mathrm{sat}}^{*} .
\end{gathered}
$$

In the last equation we have neglected the contribution of $\rho_{d \text {,dslv }}$ for the reason explained earlier in connection with Eq. (27).

Three dimensionless parameters enter the system Eqs. (37)-(40), namely,

$$
\begin{gathered}
\Lambda=-\beta_{d} \frac{\rho_{d 0}}{\rho_{a 0}-\rho_{d 0}} \\
\Theta=\frac{\pi d_{0} \rho_{\mathrm{sat}, 0} D \mathrm{Sh}_{0}}{N m_{d 0}}=\frac{6 \rho_{\mathrm{sat}, 0} D S h_{0}}{\rho_{d 0} N d_{0}^{2}} \\
V_{N}=\frac{w_{d}}{L_{n} N} .
\end{gathered}
$$

The parameter $\Lambda$ accounts for the capability of the dissolved material to provide buoyancy. For $\Lambda=1$ the soluble material provides the same amount of buoyancy whether in the disperse phase or dissolved in the ambient water, whereas there is a loss of buoyancy upon dissolution when $0<\Lambda<1$ and a gain in those fairly rare cases in which $\Lambda>1$. Negative values of $\Lambda$ signify that the dissolved material makes the ambient liquid heavier and, therefore, they amplify the loss of buoyancy associated with dissolution. For a drop plume, with the relation Eq. (50) derived in Sec. IV, $\Lambda \simeq 1$. Therefore, with that model, $\Lambda$ is not really a free parameter for drops. With $\Lambda=1$ and $V_{N}=0$, the previous system of equations reduces to the model studied in Ref. [40]. For a bubble plume, in many cases, $\beta_{d}$ is of order 1 and therefore $\Lambda \sim \rho_{d 0} / \rho_{a 0}$, which is of order 0.1 at $1000 \mathrm{~m}$ depth and smaller at shallower depths (see Table III).

The parameter $\Theta$ represents the ratio of the plume rise time, $N^{-1}$, to the time necessary for the dissolution of the $\Omega$ drops/bubbles injected per unit time. Indeed, it immediately follows from Eq. (10) that the fractions in Eq. (42) are of the order of the time necessary for the complete dissolution of a drop/bubble in a liquid containing a negligible amount of solute, for which $\rho_{d \text {, dslv }}$ can be neglected. Small $\Theta$ corresponds to a slow dissolution. In the limit $\Theta=0$, there is no dissolution at all so that $m_{d}^{*}$ remains equal to its initial value 1 . In this case the middle term in the right-hand side of the momentum Eq. (38) drops out and so does the parameter $\Lambda$. The results become therefore independent of $\Lambda$ and reduce to those presented in Ref. [7]. Conversely, dissolution is very rapid for large $\Theta$ and the plume becomes a single-phase plume.

For purposes of orientation Table I shows the values of some parameters pertinent for the Deepwater Horizon accident from Ref. [47]. 
TABLE I. Typical values of some plume parameters for the Deepwater Horizon accident calculated with $\alpha_{e}=0.11$. The buoyancy flux is defined by $Q_{d}=m_{d} / \rho_{d}=A \alpha_{d}\left(w+w_{d}\right)$ with all parameters evaluated at source conditions.

\begin{tabular}{lccccc}
\hline \hline$Q_{d} \mathrm{~m}^{4} / \mathrm{s}$ & $10^{3} N \mathrm{~s}^{-1}$ & $L_{n} \mathrm{~m}$ & $N L_{n} \mathrm{~m} / \mathrm{s}$ & $H \mathrm{~m}$ & $L_{n} / H$ \\
\hline 0.04 & 0.4 & 448 & 0.179 & 1500 & 0.299 \\
0.09 & 2.7 & 131 & 0.354 & 1500 & 0.0874 \\
\hline \hline
\end{tabular}

\section{MIXTURE LIQUID DENSITY}

One of the points made in this paper is that the dissolution of bubbles or drops may not completely remove the plume buoyancy as the dissolved material may lower the density of the liquid. In some cases as, for example, with $\mathrm{CO}_{2}$ in water, the dissolved material may actually increase the liquid density. To quantify these effects it is necessary to estimate the density of the solution. In view of the smallness of the concentration of all the dissolved quantities, as already noted, their contributions to the density superpose linearly and we can therefore limit our considerations to the effect of a single solute dissolved alone in the liquid.

Since the volume $V$ of a binary mixture is a homogeneous function of the first degree in the number of moles $n_{1}, n_{2}$ of the constituents, it follows from Euler's theorem that

$$
V=\left(\frac{\partial V}{\partial n_{1}}\right)_{p, T, n_{2}} n_{1}+\left(\frac{\partial V}{\partial n_{2}}\right)_{p, T, n_{1}} n_{2},
$$

in which $p$ and $T$ are the pressure and the (absolute) temperature. On a molar basis this relation is

$$
v=\left(\frac{\partial v}{\partial x_{1}}\right)_{p, T, x_{2}}(1-x)+\left(\frac{\partial v}{\partial x_{2}}\right)_{p, T, x_{1}} x=\bar{v}_{1}(1-x)+\bar{v}_{2} x,
$$

in which $x=n_{2} /\left(n_{1}+n_{2}\right)$ is the mole fraction of of the solute and $\bar{v}_{i}$ the partial molar volumes. If $M_{1}$ and $M_{2}$ denote the molar masses of the constituents, then the density of the solution is given by

$$
\rho=\frac{(1-x) M_{1}+x M_{2}}{(1-x) \bar{v}_{1}+x \bar{v}_{2}} \simeq \frac{M_{1}}{\bar{v}_{1}}\left[1+\frac{M_{2}}{M_{1}}\left(1-\frac{M_{1} / \bar{v}_{1}}{M_{2} / \bar{v}_{2}}\right) x,\right],
$$

with the approximation in the last step based on the assumption $x \ll 1$. Upon noting that

$$
\frac{M_{1}}{\bar{v}_{1}} \frac{M_{2}}{M_{1}} x=\frac{M_{2}}{\bar{v}_{1}} \frac{n_{2}}{n_{1}+n_{2}} \simeq \frac{m_{2}}{V},
$$

where $m_{2}$ is the dissolved mass, we may write Eq. (46) in the more convenient form

$$
\rho \simeq \frac{M_{1}}{\bar{v}_{1}}+\left(1-\frac{M_{1} / \bar{v}_{1}}{M_{2} / \bar{v}_{2}}\right) \rho_{2, \mathrm{dslv}},
$$

in which $\rho_{2, \text { dslv }}=m_{2} / V$ is the density of the dissolved material in the solution. Since $M_{1} / v_{1}=\rho_{1} \simeq$ $\rho_{a 0}$ is very close to the density of the ambient liquid, the mixture density Eq. (48) may actually be written as

$$
\rho \simeq \rho_{a 0}+\left(1-\frac{\rho_{a 0}}{M_{d} / \bar{v}_{d}}\right) \rho_{2, \mathrm{dslv}}
$$

in which $M_{d}$ and $\bar{v}_{d}$ are the molar mass and the partial molar volume of the solute. Upon comparison of Eq. (49) with Eq. (19) we thus conclude that parameter $\beta_{d}$ accounting for the change in mixture density due to dissolved material may be written as

$$
\beta_{d}=1-\frac{\rho_{a 0}}{M_{d} / \bar{v}_{d}}
$$


TABLE II. Some physical properties of a number of water-soluble liquid organic compounds at $4{ }^{\circ} \mathrm{C}$ and $10 \mathrm{MPa}$ (asterisks indicate values at standard conditions). ${ }^{a}$ Ref. [48]; ${ }^{b}$ Ref. [49]; ${ }^{c} \operatorname{Ref} .[50] ;{ }^{d} \operatorname{Ref} .[51]$; ${ }^{e}$ Ref. [52]; ${ }^{f}$ Ref. [53]; ${ }^{g}$ Ref. [54]; ${ }^{h}$ Ref. [55]; ${ }^{j}$ Ref. [56].

\begin{tabular}{llcccrr}
\hline \hline & $\begin{array}{c}\left(\rho_{d 0}\right)^{a} \\
\mathrm{~kg} / \mathrm{m}^{3}\end{array}$ & $\begin{array}{c}\left(\bar{v}_{d}^{L \infty}\right)^{b} \\
\mathrm{~cm}^{3} / \mathrm{mol}\end{array}$ & $\begin{array}{c}M_{d} / \bar{v}_{d}^{L \infty} \\
\mathrm{kg} / \mathrm{m}^{3}\end{array}$ & $\begin{array}{c}\rho_{\text {sat }, 0} \\
\mathrm{~kg} / \mathrm{m}^{3}\end{array}$ & \multicolumn{1}{c}{$\beta_{d}$} & \multicolumn{1}{c}{$\Lambda$} \\
\hline $\mathrm{CO}_{2}$ & 954 & $33.9^{*}$ & 1298 & $78.1^{g}$ & 0.245 & -5.08 \\
${\text { Ethane } \mathrm{C}_{2} \mathrm{H}_{6}}$ & 426 & $52.9^{*}$ & 563 & 10.2 & -0.877 & 0.651 \\
Propane $\mathrm{C}_{3} \mathrm{H}_{8}$ & 541 & $70.7^{*}$ & 661 & 13.1 & -0.512 & 0.603 \\
Butane $\mathrm{C}_{4} \mathrm{H}_{10}$ & 609 & $76.6^{*}$ & 757 & 15.3 & -0.321 & 0.500 \\
Ethylene $\mathrm{C}_{2} \mathrm{H}_{4}$ & 395 & $51.3^{*}$ & 545 & 22.6 & -0.834 & 0.545 \\
Benzene $\mathrm{C}_{6} \mathrm{H}_{6}$ & $879^{* h}$ & $83.3^{* c}$ & 938 & $1.88^{* h}$ & -0.0661 & 0.480 \\
Toluene $\mathrm{C}_{7} \mathrm{H}_{8}$ & 888 & $99.5^{*}$ & 929 & $0.526^{* j}$ & -0.0764 & 0.606 \\
Xylene $\mathrm{C}_{8} \mathrm{H}_{10}$ & $864^{* d}$ & $120^{* f}$ & 885 & $0.106^{* e}$ & -0.130 & -0.826 \\
\hline \hline
\end{tabular}

In an ideal mixture the components mix isometrically, i.e., with no change in the total volume, so that

$$
v^{i d}=v_{1}(1-x)+v_{2} x,
$$

with $v_{1}$ and $v_{2}$ the molar volumes of the pure constituents. The difference,

$$
v^{E}=v-v^{i d}=\bar{v}_{1}^{E}(1-x)+\bar{v}_{2}^{E} x,
$$

is the excess molar volume. From the thermodynamic relation between Gibbs's free energy and volume (see, e.g., [57]), an analogous relation between the excess free energy $g^{E}$ of each constituent and the excess volume of that constituent follows:

$$
\bar{v}_{i}^{E}=\left(\frac{\partial g_{i}^{E}}{\partial p}\right)_{T, x},
$$

or, since $g_{i}^{E}=\mathcal{R}_{G} T \log \gamma_{i}$, with $\mathcal{R}_{G}$ the universal gas constant and $\gamma_{i}$ the activity coefficient,

$$
\frac{\bar{v}_{i}^{E}}{\mathcal{R}_{G} T}=\left(\frac{\partial \log \gamma_{i}}{\partial p}\right)_{T, x} .
$$

Various approximations exist for the activity coefficients in liquid-liquid mixtures (see, e.g., Ref. [58]), but none of these includes a pressure dependence. Thus, for solutions of liquid hydrocarbons in water, one may assume that $v^{E}$ vanishes so that the rule Eq. (51) for ideal solutions applies. With this approximation, we can set $M_{d} / \bar{v}_{d}=\rho_{d 0}$ identifying the ratio with the density of the pure liquid solute and, in this case, from Eq. (50) we find

$$
\beta_{d}^{i d}=1-\frac{\rho_{a 0}}{\rho_{d 0}}
$$

With $\beta_{d}=\beta_{d}^{i d}$, it follows from the definition Eq. (41) that the parameter $\Lambda$ equals 1 . The values reported in Table II for some water-soluble liquid compounds at $4{ }^{\circ} \mathrm{C}$ and $10 \mathrm{MPa}$ show that, while $M_{d} / \bar{v}_{d}$ and $\rho_{d 0}$ are comparable, significant quantitative differences exist. In view of the great uncertainty affecting the data available in the literature, the implication of these differences remain unclear.

For a gaseous solute, since the gases of present concern are only sparingly soluble in water, we approximate the partial molar volume $\bar{v}_{d}$ by $\bar{v}_{2}^{L \infty}$, the value at infinite dilution. An empirical expression for the partial molar volume of gases dissolved in water at infinite dilution is [49]

$$
\bar{v}_{d}^{L \infty}=10.74 \mathrm{~cm}^{3} / \mathrm{mol}+0.2689 v_{c, d}^{*},
$$


TABLE III. Some physical properties of a number of water-soluble gases at standard conditions (above the line) and at $4{ }^{\circ} \mathrm{C}$ and $10 \mathrm{MPa}$ (below the line; asterisks denote values at standard conditions where appropriate values are not available); Henry's constant has been adjusted for temperature according to Ref. [59] but not for pressure. The dimensionless parameters $\beta_{d}$ and $\Lambda$ are defined in Eqs. (50) and (41), respectively. Note that, in water, methane at $4{ }^{\circ} \mathrm{C}$ and $10 \mathrm{MPa}$ may form hydrates (see, e.g., Ref. [25]). The sources for the data are: ${ }^{a}$ Ref. [48]; ${ }^{b}$ Ref. [49]; ${ }^{c}$ Ref. [59].

\begin{tabular}{|c|c|c|c|c|c|c|}
\hline & $\begin{array}{l}\left(\rho_{d 0}\right)^{a} \\
\mathrm{~kg} / \mathrm{m}^{3}\end{array}$ & $\begin{array}{c}\left(\bar{v}_{d}^{L \infty}\right)^{b} \\
\mathrm{~cm}^{3} / \mathrm{mol}\end{array}$ & $\begin{array}{c}10^{5} \text { Henry's const. } \\
\mathrm{mol} /\left(\mathrm{m}^{3} \mathrm{~Pa}\right)^{c}\end{array}$ & $\left(\rho_{\text {sat }, 0} / \rho_{d 0}\right)^{a, c}$ & $\beta_{d}$ & $10^{4} \Lambda$ \\
\hline Oxygen $\mathrm{O}_{2}$ & 1.29 & 33.2 & 1.3 & 0.0322 & -0.0375 & -0.485 \\
\hline Nitrogen $\mathrm{N}_{2}$ & 1.13 & 35.7 & 0.64 & 0.0159 & -0.275 & 3.11 \\
\hline $\mathrm{CO}_{2}$ & 1.78 & 33.9 & 33 & 0.851 & 0.230 & -4.11 \\
\hline Methane $\mathrm{CH}_{4}$ & 0.648 & 34.5 & 1.4 & 0.0347 & -1.16 & 7.49 \\
\hline Ethane $\mathrm{C}_{2} \mathrm{H}_{6}$ & 1.22 & 52.9 & 1.9 & 0.0471 & -0.763 & 9.32 \\
\hline Propane $\mathrm{C}_{3} \mathrm{H}_{8}$ & 1.83 & 70.7 & 1.5 & 0.0372 & -0.607 & 11.1 \\
\hline Butane $\mathrm{C}_{4} \mathrm{H}_{10}$ & 2.45 & 76.6 & 1.2 & 0.0297 & -0.321 & 7.88 \\
\hline Ethylene $\mathrm{C}_{2} \mathrm{H}_{4}$ & 1.15 & 51.3 & 4.8 & 0.119 & -0.832 & 9.61 \\
\hline Acetylene $\mathrm{C}_{2} \mathrm{H}_{2}$ & 1.1 & 42.5 & 41 & 1.01 & -0.635 & 6.99 \\
\hline Oxygen $\mathrm{O}_{2}$ & 150 & $33.2^{*}$ & 1.85 & 0.028 & $-0.0375^{*}$ & 66.2 \\
\hline Nitrogen $\mathrm{N}_{2}$ & 123 & $35.7^{*}$ & 0.891 & 0.014 & $-0.275^{*}$ & 386 \\
\hline Methane $\mathrm{CH}_{4}$ & 87.3 & $34.5^{*}$ & 2.10 & 0.025 & $-1.16^{*}$ & 1110 \\
\hline
\end{tabular}

in which $v_{c, d}^{*}$ is the molar critical volume of the solute. This relation represents the data with an error of about $\pm 10 \%$. Since $v_{c, d}^{*}$ is a constant, the values $\bar{v}_{d}^{L \infty}$ predicted by this relation are independent of pressure and temperature, which may be a significant limitation far from standard conditions, and partly responsible for the differences between $M_{d} / \bar{v}_{d}$ and $\rho_{d 0}$ in Table II.

Table III shows numerical values of the physical properties relevant to the present study for several gaseous solutes. The data above the horizontal line are for standard conditions $\left(25^{\circ} \mathrm{C}\right.$ and $101.3 \mathrm{kPa}$ ), at which several organic compounds are gases. The data below the line are for a temperature of $4^{\circ} \mathrm{C}$ and a pressure of $10 \mathrm{MPa}$.

\section{RESULTS: DROP PLUMES AND DEEP-WATER BUBBLE PLUMES}

The effect of the source mass and momentum fluxes on the plume dynamics have been discussed in our previous paper [7] for situations in which dissolution effects were negligible. Since the focus of the present work is on dissolution, and to limit the number of parameters, here we consider only the limit case of plumes generated by a point source, for which $m^{*}(0)=0$. We will also assume that the initial plume momentum is mostly the result of the disperse phase buoyancy, which justifies setting $M(0)=0$.

When the neutral height $L_{n}$ is much smaller than the water depth $H$, a case we refer to as deepwater, the hydrostatic effect on the bubble expansion is negligible so that $\rho_{d}^{*}=\rho_{\text {sat }}^{*}=d^{*}=1$ [see, e.g., Ref. [9]]. For the case of drops, this is true irrespective of depth due to their approximate incompressibility. This argument justifies setting $\rho_{d}^{*}=1$ in Eq. (38) and $\rho_{\text {sat }}^{*}=1$ in Eq. (40) so that the equations that we consider are

$$
\begin{gathered}
\frac{d M^{*}}{d z^{*}}=\frac{m_{d}^{*}}{w^{*}+V_{N}}+\Lambda \frac{1-m_{d}^{*}}{w^{*}}+\frac{F_{s}^{*}}{w^{*}}, \\
\frac{d m_{d}^{*}}{d z^{*}}=-\frac{\Theta \operatorname{Sh}^{*}\left(m_{d}^{*}\right)^{1 / 3}}{w^{*}+V_{N}} .
\end{gathered}
$$


With $m^{*}(0)=0, M^{*}(0)=0$, the solution of the system Eqs. (37), (39), (57), and (58) depends only on the dimensionless parameters $\Lambda, \Theta$ and $V_{N}$. The importance of these parameters has already been pointed out in Ref. [9], where parameters $G / T$ and $N / T$, related to our $\Lambda$ and $\Theta$ by $G / T=1-\Lambda$ and $N / T=1 / \Theta$, were introduced. These authors, however, did not consider the effect of the slip velocity $V_{N}$ and used $\mathrm{Sh}^{*}=d^{*}$ for the normalized Sherwood number, which implies that the dimensional mass transfer coefficient is independent of the bubble size.

\section{A. Small slip velocity}

It is convenient to start with the limit case of a very small rise velocity of the disperse phase because an approximate solution of the previous system is then available due to the fact that $V_{N}$ can be neglected in comparison with $w^{*}$. In addition to its theoretical interest, this limit is relevant for the case of a plume of small drops in a weakly stratified environment. Indeed, in order of magnitude, we have

$$
\frac{w_{d}}{w} \simeq \frac{w_{d}}{L_{n} N}=\operatorname{Re}_{d} \frac{v}{d L_{n} N}
$$

in which $\operatorname{Re}_{d}=d w_{d} / v$ is the Reynolds number of the drops-water relative motion and $v$ the water kinematic viscosity. For millimeter-size drops, $v / d \sim 10^{-3} \mathrm{~m} / \mathrm{s}$ which can be much smaller than $L_{n} N$. For example, in the Deepwater Horizon case, from Table I we see that $N L_{n} \sim 0.1 \mathrm{~m} / \mathrm{s}$. In these conditions, therefore, $w_{d} / w \ll 1$ even for $\operatorname{Re}_{d}$ of a few tens. This limit case is interesting also because the limit $w_{d} \ll w$ corresponds to the strongest coupling of the drops/bubbles with the plume water and, therefore, to the maximum buoyancy that they can impart to the plume. With this approximation, convection does not significantly contribute to the mass flux out of the drops/bubbles so that $\mathrm{Sh}=2$ and $\mathrm{Sh}^{*}=1$. This conclusion is strictly applicable only for $\operatorname{Re}_{d} \ll 1$. For larger $\operatorname{Re}_{d}$, the general dependence $\mathrm{Sh} \propto \mathrm{Re}_{d}^{1 / 2} \mathrm{Sc}^{1 / 3}$ (see Eq. (78) below) predicts a larger Sh. However, the dependence of $\mathrm{Sh}$ on $\mathrm{Re}_{d}$ remains fairly weak and keeping $\mathrm{Sh}^{*}=1$ will give results of reasonable accuracy, at least for purposes of estimation.

When $V_{N}$ is negligible compared with $w^{*}$ it is convenient to replace the variable $z^{*}$ with $m_{d}^{*}$. In this way we find

$$
\begin{gathered}
\frac{d M^{*}}{d m_{d}^{*}}=-\frac{m_{d}^{*}+\Lambda\left(1-m_{d}^{*}\right)+F_{s}^{*}}{\Theta\left(m_{d}^{*}\right)^{1 / 3}}, \\
\frac{d F_{s}^{*}}{d m_{d}^{*}}=\frac{M^{*}}{\Theta\left(m_{d}^{*}\right)^{1 / 3}} .
\end{gathered}
$$

These two equations form a closed system to be solved subject to the condition $F_{s}^{*}=0$ at the source, where $m_{d}^{*}=1$. The solution for general $M^{*}$ at the source is complicated, but some insight can be gained by considering the solution for the special initial condition $M^{*}=0$ which, as noted before, attributes the entire plume momentum to the buoyancy of the disperse phase. With $M^{*}=0$ the solution of Eqs. (60) and (61) is

$$
\begin{aligned}
M^{*}= & \sin \left(\frac{3\left(1-\left(m_{d}^{*}\right)^{2 / 3}\right)}{2 \Theta}\right)+(\Lambda-1) \Theta\left\{\left(m_{d}^{*}\right)^{1 / 3}-\cos \left(\frac{3\left(1-\left(m_{d}^{*}\right)^{2 / 3}\right)}{2 \Theta}\right)\right. \\
+ & \left.\sqrt{\frac{\pi \Theta}{3}}\left[\cos \left(\frac{3\left(m_{d}^{*}\right)^{2 / 3}}{2 \Theta}\right) I_{C}+\sin \left(\frac{3\left(m_{d}^{*}\right)^{2 / 3}}{2 \Theta}\right) I_{S}\right]\right\}, \\
F_{s}^{*}= & \cos \left(\frac{3\left(1-\left(m_{d}^{*}\right)^{2 / 3}\right)}{2 \Theta}\right)+(\Lambda-1) m_{d}^{*}-\Lambda \\
& +(\Lambda-1) \sqrt{\frac{\pi \Theta^{3}}{3}}\left[\sin \left(\frac{3\left(m_{d}^{*}\right)^{2 / 3}}{2 \Theta}\right) I_{C}-\cos \left(\frac{3\left(m_{d}^{*}\right)^{2 / 3}}{2 \Theta}\right) I_{S}\right],
\end{aligned}
$$


in which

$$
I_{C}=\sqrt{\frac{2}{\pi}} \int_{\sqrt{3 /(\pi \Theta)\left(m_{d}^{*}\right)^{1 / 3}}}^{\sqrt{3 /(\pi \Theta)}} \cos \eta^{2} d \eta, \quad I_{S}=\sqrt{\frac{2}{\pi}} \int_{\sqrt{3 /(\pi \Theta)\left(m_{d}^{*}\right)^{1 / 3}}}^{\sqrt{3 /(\pi \Theta)}} \sin \eta^{2} d \eta
$$

can be expressed in terms of Fresnel's integrals. With these results, $z^{*}$ and $m^{*}$ can be found by integrating

$$
\begin{aligned}
\frac{d m^{*}}{d m_{d}^{*}} & =-\frac{\left(M^{*}\right)^{3 / 2}}{\Theta m_{d}^{*} m^{*}} \\
\frac{d z^{*}}{d m_{d}^{*}} & =-\frac{M^{*}}{\Theta m_{d}^{*} m^{*}}
\end{aligned}
$$

subject to $z^{*}=0$ and $m^{*}=0$ for $m_{d}^{*}=1$. We do not show these expressions for brevity.

The previous solution simplifies considerably in the case $\Lambda=1$ which, as noted before, is approximately applicable for drops:

$$
\begin{gathered}
M^{*}=\sin \left(\frac{3\left(1-\left(m_{d}^{*}\right)^{2 / 3}\right)}{2 \Theta}\right), \\
F_{s}^{*}=\cos \left(\frac{3\left(1-\left(m_{d}^{*}\right)^{2 / 3}\right)}{2 \Theta}\right)-1 .
\end{gathered}
$$

In this case the plume momentum vanishes at the peel height, where the argument of the sine in Eq. (67) equals $\pi$ :

$$
\left(m_{d}^{*}\right)^{2 / 3}=1-\frac{2 \pi}{3} \Theta
$$

Since $0 \leqslant m_{d}^{*}$, the plume completely dissolves before reaching the peel height when $\Theta>3 /(2 \pi) \simeq$ 0.4775 . The value $\Theta=3 /(2 \pi)$ identifies the critical situation in which the soluble material is completely dissolved right at the peel height. When $\Theta<3 /(2 \pi)$, enough of the soluble disperse phase is left to give rise to a second plume, which will be characterized by a larger value of $\Theta$ in view of the partial dissolution of the drops/bubbles that has already taken place. This process will repeat itself until $\Theta$ exceeds the critical value. The neutral height corresponds to the vanishing of the right-hand side of Eq. (60) and is found therefore to correspond to

$$
\left(m_{d}^{*}\right)^{2 / 3}=1-\frac{\pi}{3} \Theta .
$$

The soluble phase will have totally dissolved at the neutral height for $\Theta=3 / \pi$.

For a general value of $\Lambda \neq 1$, these critical situations-namely, total dissolution at the peel height or at the neutral height—are encountered, respectively, when the following relations between $\Lambda$ and $\Theta$ hold:

$$
\Lambda=1-\frac{\sin \left(\frac{3}{2 \Theta}\right)}{\sqrt{\frac{\pi \Theta^{3}}{3}} C\left(\frac{3}{\pi \Theta}\right)-\Theta \cos \left(\frac{3}{2 \Theta}\right)},
$$

and

$$
\Lambda=1-\frac{\cos \left(\frac{3}{2 \Theta}\right)}{\Theta \sin \left(\frac{3}{2 \Theta}\right)-\sqrt{\frac{\pi \Theta^{3}}{3}} S\left(\frac{3}{\pi \Theta}\right)},
$$

in which $C$ and $S$ are the cosine and sine Fresnel integrals. Graphs of these two lines in the $\Lambda, \Theta$ plane are shown in Fig. 1. For large $\Theta$ dissolution is rapid and, in order for it to be complete at the peel or neutral height, the plume has to slow down rapidly. This requires negative value of $\Lambda$ for the 


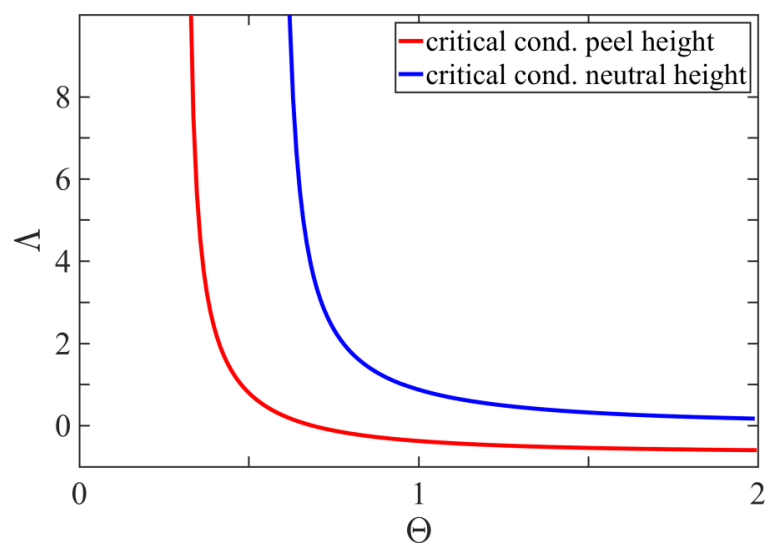

FIG. 1. Relation between the parameters $\Lambda$ and $\Theta$, defined in Eqs. (41) and (42), respectively, corresponding to total dissolution of the disperse phase at the neutral height (upper curve) and at the peel height. Below the curves the disperse phase does not dissolve completely before reaching the neutral or peel height. A second rising plume will be formed, with a smaller value of $\Theta$ and so forth until $\Theta$ becomes so small that the disperse phase dissolves below the last peel height.

peel height and a small value of $\Lambda$ for the neutral height. A series expansion for large $\Theta$ produces the asymptotic result

$$
\Lambda=-\frac{2}{3}+\frac{5}{18 \Theta^{2}}+\frac{41}{2808 \Theta^{4}}+O\left(\Theta^{-9 / 2}\right)
$$

for the peel height, and

$$
\Lambda=\frac{9}{14 \Theta^{2}}+\frac{675}{4312 \Theta^{4}}+O\left(\Theta^{-5}\right)
$$

for the neutral height. Conversely, for small $\Theta$, dissolution is very slow and, in order for it to be completed at the peel or neutral height, these heights must be large, which requires the plume buoyancy to increase with dissolution and the value of $\Lambda$ to increase.

Figure 2 shows some numerical results for the peel and neutral heights for this case in which $V_{N} \ll w$. The left panel shows the dependence of $h_{p}$ (red) and $h_{n}$ (blue) on $\Lambda$ for $\Theta=0$ (solid), 1 (dashed), and 4 (dash-dots). The right panel shows the dependence of $h_{p}$ and $h_{n}$ on $\Theta$ for $\Lambda=1.5$ (solid), 1 (dashed) and -0.5 (dash-dots). For an insoluble disperse phase, $\Theta=0$ and $\Lambda$ has no effect on the plume, which is shown by the horizontal lines in the left panel of the figure, with the peel height $h_{p}=2.6$ [40] as also found in our earlier paper [7]. A comparison with the other curves shows the importance of dissolution. When the parameter $\Theta$ increases past 1 , however, dissolution is so rapid that the results become essentially independent of $\Theta$. With $\Lambda=1$ the loss of buoyancy caused by the dissolution of the soluble component is exactly balanced by the equal gain in buoyancy of the ambient liquid due to the dissolved material. In this case, with $V_{N}$ negligible compared to $w^{*}$, $m_{b}^{*}$ has no influence on $M^{*}$, which implies that the peel height and neutral height are independent of the parameter $\Theta$. Indeed, in Fig. 2, the curves corresponding to different $\Theta$ all cross at the same point when $\Lambda=1$.

More generally, the total plume buoyancy increases with $\Lambda$, as the loss of buoyancy is mitigated by the lightening of the solution (for $0<\Lambda<1$ ) or even over-compensated (for $1<\Lambda$ ). In these circumstances dissolution is beneficial and the peel and neutral heights increase with $\Theta$ as can be seen in the right panel of Fig. 2 for the case $\Lambda=1.5$. The effect, however, is only appreciable over a limited range for small $\Theta$ as the dissolution rate increases with $\Theta$. As this parameter increases, dissolution occurs closer and closer to the source, the two-phase plume becomes effectively a singlephase plume and the total plume buoyancy comes to depend only on the parameter $\Lambda$. Conversely, 

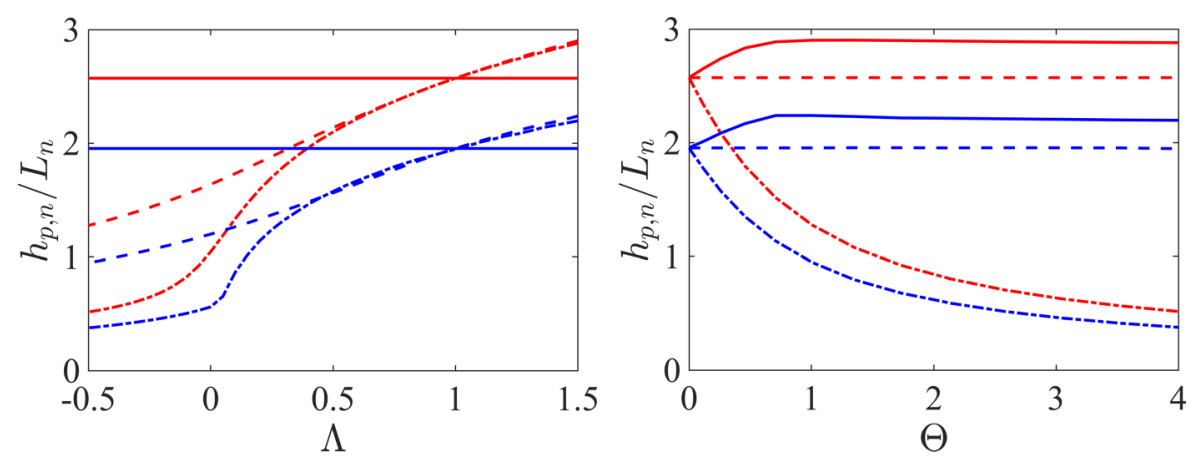

FIG. 2. Left: Dependence of the normalized peel height $h_{p}$ (red curves) and neutral height $h_{n}$ (blue curves) upon the parameter $\Lambda$ for negligible slip velocity, $V_{N}=0$. The solid, dashed, and dash-dotted lines are for $\Theta=0,1,4$, respectively. Right: Dependence of $h_{p}$ (red curves) and $h_{n}$ (blue curves) on the parameter $\Theta$ for $\Lambda=1.5$ (solid) 1 (dashed), and -0.5 (dash-dotted). The parameter $\Lambda$, defined in Eq. (41), describes the effect of the dissolved material on the density of the plume liquid. The parameter $\Theta$, defined in Eq. (42), accounts for the relative rapidity of dissolution as compared with the plume ascent time; large $\Theta$ implies rapid dissolution.

for $\Lambda<1$, the dissolved component provides less buoyancy than the discrete component. Therefore, rapid dissolution (larger $\Theta$ ) will decrease the total plume buoyancy and thus decrease the plume peel and neutral heights. Consequently, $h_{p}$ and $h_{n}$ decrease with $\Theta$.

\section{B. Finite slip velocity}

When the slip velocity cannot be neglected, the exploration of the parameter space and the presentation of results becomes more difficult. To begin with, it is necessary to deal with the large number of different correlations available in the literature for the drag coefficient and Sherwood number for translating drops/bubbles. Secondly, the explicit dependence of the Reynolds and Sherwood numbers on the size of the drops/bubbles and their physical properties increases considerably the size of the parameter space. Since it is impossible to present a complete exploration of this space, here we limit ourselves to some illustrative results. We focus mostly on the case of bubbles.

A widely used correlation for the bubble slip velocity has been given in Ref. [60]:

$$
\frac{w_{b}}{w_{c}}=0.0545 F^{3 / 4} \frac{\left(1+1.31 \times 10^{-5} \mathrm{Mo}^{11 / 20} F^{73 / 33}\right)^{21 / 176}}{\left(1+0.02 F^{10 / 11}\right)^{10 / 11}},
$$

where Mo $=g \mu^{4} /\left(\rho \sigma^{3}\right)$ is the Morton number,

$$
F=g\left(\frac{\rho^{5} d^{8}}{\sigma \mu^{4}}\right)^{1 / 3}
$$

is the so-called flow number, and

$$
w_{c}=1.53\left(\frac{\sigma g\left(\rho-\rho_{d}\right)}{\rho^{2}}\right)^{1 / 4}
$$

is a velocity scale close to that in the plateau region for the slip velocity of bubbles in small-Mo fluids (see, e.g., Refs. [30,61]). The advantage of this correlation is that it gives the bubble rise velocity explicitly with no need for iteration. For the Sherwood number we use the classic relation of Ref. [62] developed for contaminated bubbles with a no-slip interface:

$$
\mathrm{Sh}=2+0.95 \mathrm{Re}_{d}^{1 / 2} \mathrm{Sc}^{1 / 3} .
$$



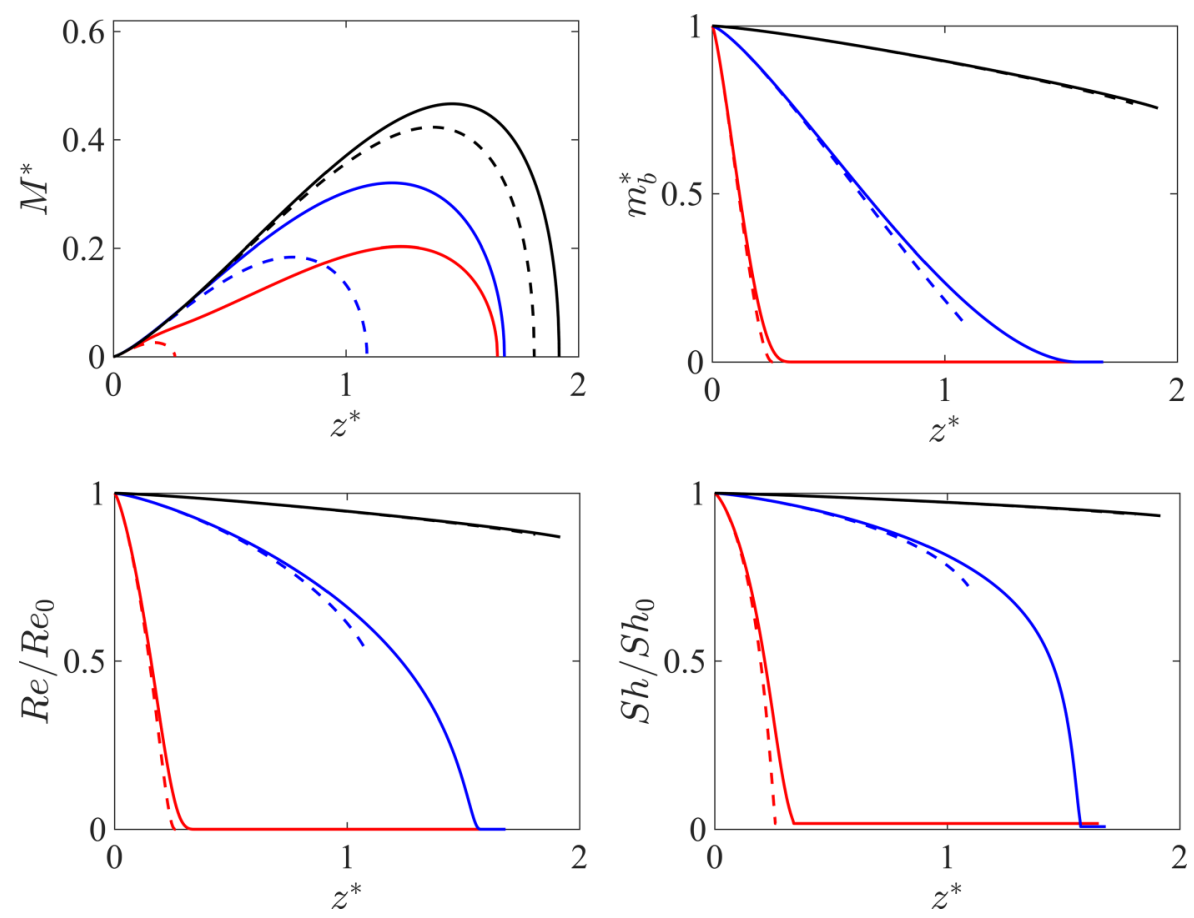

FIG. 3. Dependence on the normalized height $z^{*}=z / L_{n}$ above the source of the normalized plume momentum flow rate $M^{*}$, disperse-phase mass flow rate $m_{d}^{*}$, and bubble Reynolds and Sherwood numbers normalized by their initial values, $\mathrm{Re}_{d} / \mathrm{Re}_{d 0}$ and $\mathrm{Sh} / \mathrm{Sh}_{0}$. The red, blue, and black curves are for $\left(\Theta, V_{N}\right)=(19.2,0.63),(2.1,0.69)$, and $(0.26,0.92)$, respectively. For the conditions specified in the text the corresponding values of the initial dimensional bubble diameter are $d=1.00,4.47$, and $20.0 \mathrm{~mm}$. Solid and dashed lines are for $\Lambda=0.2$ and -0.2 , respectively.

In using this correlation to calculate the mass transfer coefficient, we neglect the area increase of the bubbles due to their deformation, which usually is a small effect [see, e.g., Ref. [30], p. 194]; it could be included, if desired, by introducing a shape factor $[63,64]$. It may be noted that Sh and $w_{d}$ and, therefore, their normalized counterparts $\mathrm{Sh}^{*}$ and $V_{N}$, evolve in the course of the simulation due to the decrease of the diameter $d$ upon dissolution. Some representative results for the normalized plume momentum flow rate $M^{*}$, bubble mass flow rate $m_{d}^{*}$, and the drop/bubble Reynolds and Sherwood numbers normalized by their initial values, $\mathrm{Re} / \mathrm{Re}_{0}$ and $\mathrm{Sh} / \mathrm{Sh}_{0}$, all versus the normalized depth $z^{*}=z / L_{n}$, are shown in Fig. 3. For $D=1.49 \times 10^{-9} \mathrm{~m}^{2} / \mathrm{s}$, the physical properties of methane, and the conditions of the Deepwater Horizon accident, with $N=2.7 \times 10^{-3}$ s, with a water column depth of $1500 \mathrm{~m}$ (Table I), these correspond to initial bubble sizes between 1 and $20 \mathrm{~mm}$; the lines in each family correspond to two different values of $\Lambda, \Lambda=0.2$ (solid) and $\Lambda=-0.2$ (dashed); these values are fairly large for most gases, but serve to bracket the range of the phenomena observed. The red, blue and black curves are for $\left(\Theta, V_{N}\right)=(19.2,0.63),(2.1,0.69),(0.26,0.92)$, corresponding to initial bubble diameters $d(0)$ of $1.00,4.47$ and $20.0 \mathrm{~mm}$, respectively, injected with the same volume flow rate of $0.09 \mathrm{~m}^{3} / \mathrm{s}$ with $N L_{n}=0.343 \mathrm{~m} / \mathrm{s}$.

The first panel shows the plume momentum, which is a maximum at the neutral height and vanishes at the peel height. Since the strength of the liquid-bubbles coupling increases as the rise velocity and, therefore, the bubble radius, decreases, one would expect that, for the same gas injection rate, the buoyancy effect would be strongest for the smallest bubbles. The rate of dissolution, however, tends to oppose this tendency. Indeed, in the figure we see that the peel height (where $M^{*}=0$ ) for $\Lambda=0.2$ is lowest for the smallest bubbles and increases with bubble 
size, following an inverse trend with the dissolution rate (decreasing $\Theta$ ). That is, the faster the dissolution rate, the lower the peel height, in inverse order of the strength of the bubbles-liquid momentum coupling. As expected, this outcome is even starker for $\Lambda=-0.2$ as, in this case, dissolution makes the liquid heavier (a typical case being $\mathrm{CO}_{2}$ ) and further diminishes the effect of buoyancy. The effect is minor when the dissolution rate is slow as shown by the closeness of the black solid and dashed curves.

Interestingly, while the neutral heights (the maxima of the curves in the first panel) are significantly larger for $\Theta=2.1$ (blue, $d=4.47 \mathrm{~mm}$ ) than for $\Theta=19.2$ (red, $d=1 \mathrm{~mm}$ ), the final peel heights are very close. The reason is that, below the neutral height, the $4.47 \mathrm{~mm}$ bubbles impart to the plume a larger momentum than the smaller bubbles due to their slower dissolution. With the larger momentum, however, comes a larger entrainment of the relatively heavier deep liquid so that, ultimately, both peel heights turn out to be very similar. Generally speaking, while an increased mass flow rate always decreases the neutral and peel heights due to the increased entrainment [the right-hand side of Eq. (37)], the momentum flow rate has a twofold effect, as in this case, and as implied by the opposite signs between the first and last terms in the right-hand side of Eq. (38).

The effect of $\Lambda$ on $m_{b}^{*}, \operatorname{Re}_{d}$ and $\mathrm{Sh}$ is minor. The smaller bubbles dissolve completely before reaching the neutral height (second panel in Fig. 3), but buoyancy is not completely lost when $\Lambda=0.2$ and only modestly decreased when $\Lambda=-0.2$. Their normalized Reynolds number also decreases rapidly [Fig. 3(c)] and the Sherwood number Sh tends to 2, so that $\mathrm{Sh}^{*} \rightarrow 2 / \mathrm{Sh}_{0}$ [Fig. 3(d)]. Due to their slow dissolution rate, for the largest bubbles the difference between the two values of $\Lambda$ is barely discernible as far as $m_{d}^{*}$, $\mathrm{Re}_{d}$ and $\mathrm{Sh}$ are concerned and manifests itself mostly in the height dependence of $M^{*}$. Of the three bubble sizes considered here, only the largest ones (black lines) will persist beyond the peel height and will give rise to one or more repeated plumes above this level.

It is well-known that the rise velocity of bubbles in water takes on an approximately constant value, close to Eq. (77), for equivalent bubble diameters in the range between about 1 and $30 \mathrm{~mm}$ (see, e.g., Ref. [30]). Once larger bubbles dissolve to diameters below $1 \mathrm{~mm}$, they have lost most of their initial amount of gas and the subsequent dissolution is very rapid as shown by the red lines in the lower two panels of Fig. 3. Thus, it makes sense to focus on the period of the bubble life when their radius is larger than $1 \mathrm{~mm}$ and their drift velocity approximately constant. For these bubbles, fixing $V_{N}$ at its initial source value will result in a relatively small error. The same approximation is likely to incur a small error for bubbles larger than $30 \mathrm{~mm}$ as well, as their dissolution will not have progressed very much by the time they reach the peel height. The Sherwood number is dependent on $\mathrm{Re}_{d}$ rather than on the drift velocity, which involves the bubble diameter and, therefore, a greater sensitivity to the bubble size. Nevertheless, the blue and black lines in the last panel of Fig. 3 indicate that the approximation $\mathrm{Sh} \sim$ constant, followed by a very rapid decline to zero, captures the essence of the Sherwood number dependence on height. These considerations justify considering the parameters $V_{N}$ and Sh constant as we continue our parameter study in the next section.

The rise velocity of drops in an immiscible liquid also exhibits a plateau as a function of the equivalent diameter similarly to gas bubbles (see, e.g., Ref. [30]), characterized by a velocity of the order of $w_{c}$ defined in Eq. (77). For such drops, with sizes of a few millimeters or larger, the Reynolds number $d w_{c} / v$ is typically of the order of a few hundreds. With Sc $\sim 10^{3}$, the Sherwood number given by Eq. (78) becomes of the order of 100. According to the data of Table II, the ratio $\rho_{\text {sat }, 0} / \rho_{d 0}$ appearing in the definition of the parameter $\Theta$ in Eq. (42) is, however, of the order of $10^{-3}-10^{-2}$, as opposed to the order of $10^{-2}$ or larger for bubbles. As a consequence, the typical values of $\Theta$ tend to be smaller than for the case of bubbles so that these drops dissolve slowly. We are thus led to a situation in which the results of our previous paper [7], in which dissolution was neglected, and those of the next subsection, in which $V_{N}$ is assumed to be constant, become approximately applicable. Thus, we feel justified in omitting a detailed study of this case. 


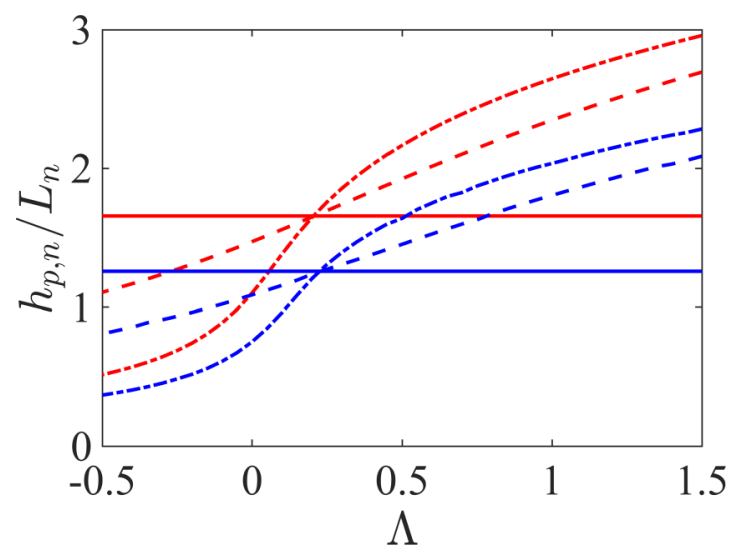

FIG. 4. Dependence of the normalized peel height $h_{p}$ (red curves) and neutral height $h_{n}$ (blue curves) upon the parameter $\Lambda$ for a normalized slip velocity $V_{N}=2$. The solid, dashed, dash-dotted lines are for $\Theta=0,1,4$, respectively.

\section{Constant $V_{N}$ and Sh}

With $V_{N}=V_{N}(0)$ constant and $\mathrm{Sh}^{*} \simeq 1$, the behavior of the present system comes to depend on the three parameters $V_{N}, \Lambda$ and $\Theta$. As noted in our earlier paper [7], the slip velocity $V_{N}$ is large when the bubbles are weakly coupled to the liquid and are therefore unable to impart much momentum to it. In this case the effect of the bubble buoyancy flux is weak with the consequence that the peel height is small. This trend is evident from the right-hand side of Eq. (57) in which the influence of the first term, which is the dominant driving term, decreases with increasing $V_{N}$.

Figure 4 shows the dependence of $h_{p}$ and $h_{n}$ on $\Lambda$ for $V_{N}=2$ and different values of $\Theta$. For $\Theta=0, m_{b}^{*}$ remains equal to 1 , and therefore, the parameter $\Lambda$ in Eq. (57) becomes irrelevant. Thus, the corresponding curves are just straight horizontal lines. For $\Theta>0$, as in the previous case of Fig. 2, in this case also we see the strong effect of $\Lambda$ with both $h_{p}$ and $h_{n}$ increasing functions of this parameter.

One notices in this figure that the lines corresponding to different values of $\Theta$ appear to go through a single point $\tilde{\Lambda}$. We mentioned a special case of this result in Sec. V A where, for $V_{N} \ll w^{*}$, we saw that lines for different $\Theta$ 's all cross at $\Lambda=1$. When $V_{N}$ is not small, as in the case of this figure, this special value of $\Lambda$ comes to depend on $V_{N}$. A detailed analysis of the numerical results shows that, in fact, a very weak dependence on $\Theta$ is always present, although the effect is very small for values of $V_{N}$ and $\Lambda$ in particular small ranges about values $\tilde{V}_{N}$ and $\tilde{\Lambda}$ as can be observed in this figure. We can understand this result by focusing on the first and second terms in the right-hand side of the momentum Eq. (57):

$$
\frac{m_{d}^{*}}{w^{*}+V_{N}}+\Lambda \frac{1-m_{d}^{*}}{w^{*}}
$$

Both these terms correspond to buoyancy sources: The first one is the buoyancy provided by the rising drops/bubbles, the second one is the buoyancy provided by the dissolved material. With $\Lambda=1$ and $V_{N}=0$, the decrease of buoyancy caused by dissolution is exactly balanced by the increase provided by the lightening of the plume liquid. In these conditions, the rate of change of $m_{d}^{*}$ and, with it, the influence of the parameter $\Theta$, becomes irrelevant. When $0<\Lambda<1$, however, the second effect is reduced. For the two terms to approximately balance so as to add up to an effect approximately independent of $\Theta$, it is therefore necessary for the first term to be reduced as well and, therefore, for $V_{N}$ to increase. A telltale sign of the approximate nature of this balance is that the value of $\Lambda$ at which the lines corresponding to different $\Theta$ 's appear to intersect is slightly different 

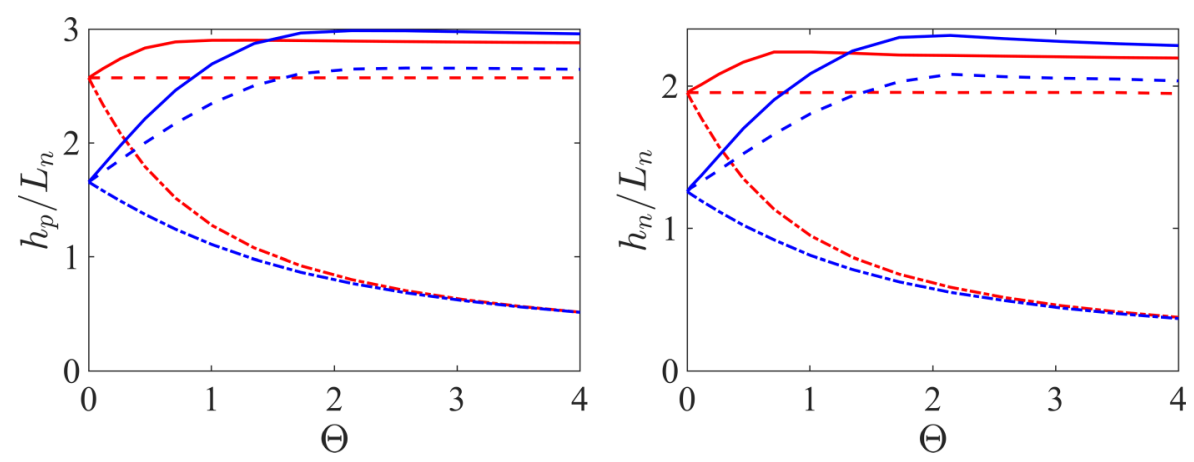

FIG. 5. Dependence of the normalized peel height $h_{p}$ (left) and neutral height (right) on the parameter $\Theta$ for $\Lambda=1.5$ (solid), 1 (dashed), and -0.5 (dash-dotted). The red curves are for $V_{N}=0$, the blue curves for $V_{N}=2$, both held constant.

for $h_{n}$ and $h_{p}$. Figure 4 shows that both $h_{p}$ and $h_{n}$ increase with $\Theta$ for $\Lambda$ to the right of $\tilde{\Lambda}$, while they tend to decrease with $\Theta$ for $\Lambda$ to the left of it.

Figure 5 shows the dependence of $h_{p}$ and $h_{n}$ on $\Theta$ for different $\Lambda$ and $V_{N}=0$ (red curves) and 2 (blue curves). For $\Theta=0$, i.e., no dissolution, the density of the plume liquid is unaffected and therefore the curves corresponding to different $\Lambda$ 's start at the same point. For $\Lambda=1.5$ (solid lines), both $h_{p}$ and $h_{n}$ are initially increasing functions of $\Theta$ because, since $\Lambda>1$ implies a gain of buoyancy upon dissolution, a faster dissolution increases the plume momentum. At some point, however, the dissolution becomes so fast compared with the rise time of the plume that further increases are immaterial. It is found that, by increasing $\Theta$ beyond the range shown in the figure, the curves corresponding to $V_{N}=0$ and $V_{N}=2$ come together. Complete dissolution occurs fast enough that the two-phase plume becomes essentially a single-phase plume with a liquid lighter than the ambient and $\Theta$ ceases to influence the results. In these conditions the analysis of Ref. [40] applies.

For $\Lambda=1$ (dashed lines in Fig. 5) there is no effect of $\Theta$ for $V_{N}=0$, as already noted in connection with Fig. 4, while an initial growing trend with $\Theta$ is found for $V_{N}=2$. This effect occurs because, as long as the dissolving material remains in the drops/bubbles, its effect on buoyancy is limited by the nonzero value of $V_{N}$ as explained before. However, when the material enters into solution, the drag exerted by rising drops/bubbles [first term in Eq. (79)] is replaced by a lightening of the plume liquid [second term in Eq. (79)], which is more effective. As before, the curves come together for $\Theta$ beyond the range shown in the figure.

For $\Lambda=-0.5$ (dash-dotted lines) the solution is heavier than the pure liquid and, the faster the dissolution, the lower the neutral and peel heights. In this case, for $\Theta$ large, once again we have effectively a single-phase plume and the effect of $V_{N}$ disappears. In this case the confluence of the curves for different $V_{N}$ 's occurs for smaller values of $\Theta$ than for positive $\Lambda$.

A direct comparison of the results for constant and variable $V_{N}$ and Sh is shown in Fig. 6. The solid and dashed curves, for $h_{p}$ and $h_{n}$, respectively, show results for $V_{N}$ and Sh calculated from Eqs. (75) and (78) with the instantaneous value of the bubble diameter. The dash-dotted lines are for $h_{p}$ and $h_{n}$ calculated keeping the rise velocity and Sherwood number constant at their values at the source. The negligible difference between solid and dashed lines over the majority of the parameter range studied justifies the approximation of keeping $V_{N}$ and $S h$ constant at their source values.

When the drops/bubble totally dissolve the nature of the plume changes from two-phase to single-phase and, in the dilute limit that we consider, the further rise of the plume is severely limited. It is therefore interesting to investigate the height $h_{\mathrm{dslv}}$ at which the drops/bubbles completely dissolve as a function of the model parameters. These results are shown in Figs. 7, 8, and 9; in these figures the curves begin where the complete dissolution height equals the peel height. Figure 7 shows 

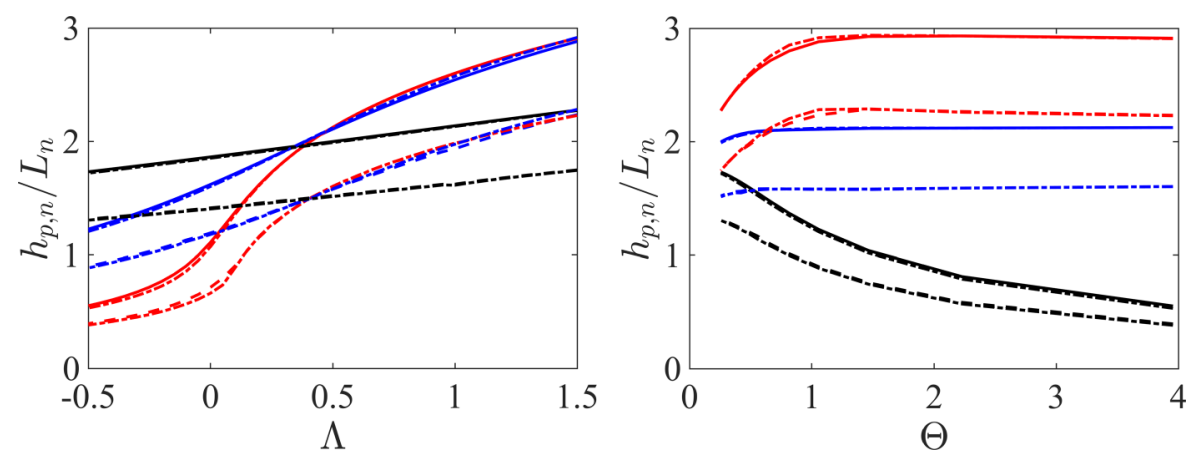

FIG. 6. Peel height $h_{p}$ (solid lines) and neutral heigh $h_{n}$ (dashed lines) as predicted with size-dependent rise velocity and Sherwood number compared with predictions based on values of rise velocity and Sherwood number held constant at the source level (dash-dotted lines). In the left panel the red, blue, black curves are for $\Theta=4,1,0.26$, respectively. In the right panel the red, blue, black curves are for $\Lambda=1.5,1,-0.5$, respectively. The black dash-dotted line in the left panel and the blue and black lines in the right panel are actually a superposition of the dashed and dash-dotted lines. The predictions for $h_{n}$ in these cases are indistinguishable within the thickness of the lines.

the normalized total dissolution height as a function of $\Theta$ for $V_{N}=0,1,2$, and 4 with $\Lambda=1$. As expected, the dissolution height rapidly decreases with increasing dissolution rate $\Theta$ and increases with the drop/bubble rise velocity $V_{N}$. The effect of the drop/bubble rise velocity is shown in Fig. 8 . The upper group of lines is for $\Theta=2$ and the lower one for $\Theta=4$; in each group, in ascending order, the lines are for $\Lambda=0,0.5,1$, and 1.5. The expected upward trend with increasing $V_{N}$ of the previous figure is confirmed. As $\Lambda$ increases the loss of buoyancy due to dissolution is mitigated and the dissolution height correspondingly increases. The dependence of the dissolution height on $\Lambda$ is weak, but is strongly affected by the increasing peel height with increasing $\Lambda$. The effect of the dissolution rate parameter $\Theta$, however, is marked as seen before. The relatively small effect of the parameter $\Lambda$ is clearer in Fig. 9 in which the curves are ordered according to increasing $V_{N}$ and $\Theta$. For negative values of $\Lambda$ dissolution magnifies the loss of buoyancy and complete dissolution occurs at such small heights that they are not visible in graphs of this type.

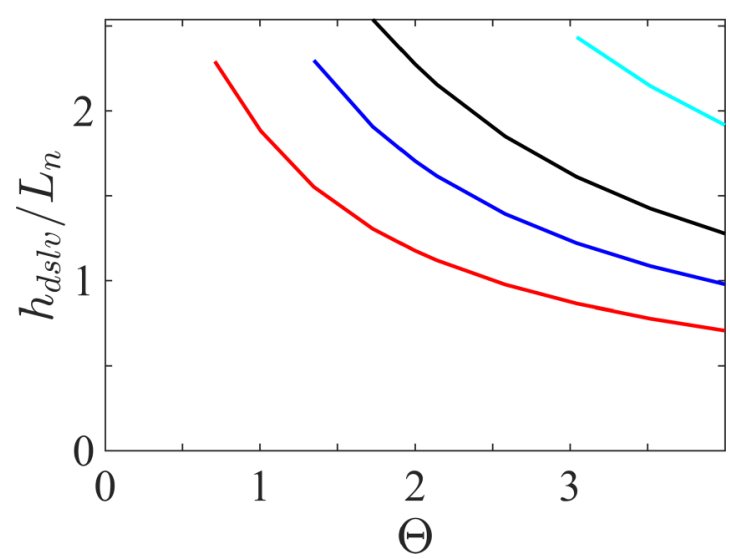

FIG. 7. Normalized height for complete dissolution for $\Lambda=1$, in which case there is no loss of buoyancy with dissolution. In ascending order, the curves are for dimensionless drop/bubble rise velocity $V_{N}=0,1,2$, and 4 . The curves begin at the value of the dissolution rate parameter $\Theta$ corresponding to the peel height. 


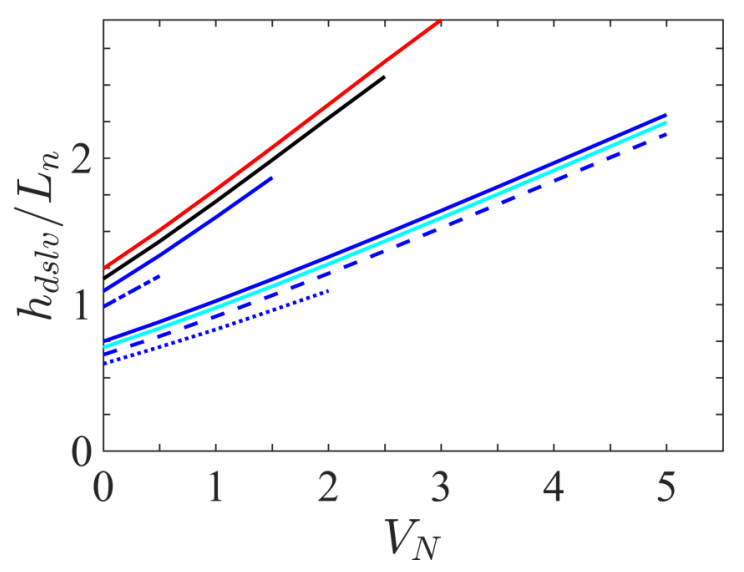

FIG. 8. Normalized height for complete dissolution as function of the dimensionless drop/bubble rise velocity $V_{N}$; the upper group of lines is for $\Theta=2$ and the lower one for $\Theta=4$; in each group, in ascending order, the lines are for $\Lambda=0,0.5,1$, and 1.5 .

\section{RESULTS: BUBBLE PLUMES IN FINITE WATER DEPTH}

At shallow water depths, it may happen that the (theoretical) peel height is larger than the liquid depth so that no intrusion can form except at the surface. Since the peel and neutral heights have a comparable magnitude, a comparison of the water depth with the characteristic length $L_{n}$ defined in Eq. (33) may be used as an approximate guide to decide whether this is a possibility in any specific case. As Eq. (33) shows, $L_{n}$ depends fairly strongly on the degree of stratification, decreasing when stratification increases.

Other than for this aspect, there are two important reasons why the previous model needs to be modified for shallow submergence of the plume source, especially for a bubble plume. In the first place, the solubility of gases has a strong dependence on pressure, which may vary significantly as the bubbles rise. Second, the falling hydrostatic pressure will cause the bubbles to expand. These factors are less of a concern for a drop plume in view of the near-incompressibility of most liquids

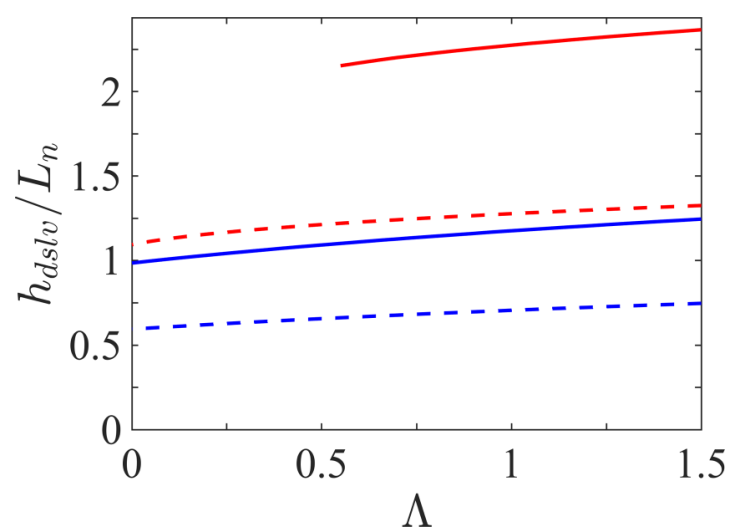

FIG. 9. Normalized height for complete dissolution as function of the parameter $\Lambda$. In ascending order the curves are for $V_{N}=0, \Theta=4 ; V_{N}=0, \Theta=2 ; V_{N}=2, \Theta=4$; and $V_{N}=2, \Theta=2$. The topmost line begins where the complete dissolution height equals the peel height. For negative values of $\Lambda$ dissolution magnifies the loss of buoyancy and complete dissolution occurs at such small heights that they are not visible in graphs of this type. 
and of the small effect of pressure on liquid-liquid solubilities. For these reasons, in this section we focus on bubble plumes. Since we are dealing with limited depths, the gas density will be orders of magnitude smaller than the liquid density so that the parameter $\Lambda$ defined in Eq. (41) can be taken as zero.

The bubble volume is determined by two countervailing effects, gas dissolution and expansion due to the pressure falling with height. When the latter dominates, the bubbles tend to rise relatively fast, their coupling with the liquid is reduced and both the peel and neutral height tend to decrease. When gas dissolution is the dominant effect, buoyancy is quickly lost and the peel and neutral height will also decrease. Thus, for shallow plumes, one may expect the existence of optimal conditions such the the two effects are in balance and the peel and neutral heights reach a maximum.

The compressibility factor $Z$ for a gas of molecular mass $M$ occupying a volume $V$ at pressure $p$ and temperature $T$ is defined by

$$
Z=\frac{M p}{\mathcal{R}_{G} \rho_{d} T},
$$

in which $\mathcal{R}_{G}$ is the universal gas constant and we write $\rho_{d}$ for the gas density consistently with the notation used in the previous sections. Since $Z$ is usually a fairly weak function of $p$ over a range of a few atmospheres, we have, approximately,

$$
\rho_{d}^{*}=\frac{\rho_{d}}{\rho_{d 0}} \simeq \frac{p}{p_{0}}=p^{*}
$$

For the effect of pressure on solubility we use Henry's law assuming a direct proportionality to the local pressure. As a consequence, the ratio $\rho_{\text {sat }, 0} / \rho_{d 0}$ in the definition Eq. (42) of the parameter $\Theta$ is independent of the water depth so that $\Theta$ itself is also independent of the water depth.

The pressure $p_{0}$ at the source level $z=0$ is the sum of the atmospheric pressure $p_{\text {atm }}$ and the hydrostatic pressure, which we write as $\rho_{a} g H$, in which $H$ is the depth of the source and the water density can be taken as constant even in the presence of stratification with negligible error. Thus, we may write

$$
p^{*}=1-\frac{\rho_{a} g z}{p_{\mathrm{atm}}+\rho_{a} g H}=1-\frac{L_{n}}{H_{t}} z^{*},
$$

where $z$ is measured upward from the source level as before and $H_{t}=H+p_{\mathrm{atm}} /\left(\rho_{a} g\right)$, with $p_{\text {atm }} /\left(\rho_{a} g\right)=H_{a} \simeq 10 \mathrm{~m}$, is the total head at the source. The parameter $L_{n} / H_{t}$ determines the relative importance of the water depth in affecting the expansion of the rising bubbles. The case addressed in the previous section can be considered as the limit in which $L_{n} / H_{t} \ll 1$.

Another point to keep in mind is that the peel height has an upper limit given by the water depth $H$ as mentioned before. Thus, it is necessary that $h_{p} \leqslant H$ or

$$
\frac{h_{p}}{L_{n}} \leqslant \frac{H_{t}}{L_{n}} \frac{H}{H_{a}+H},
$$

with $h_{p} / L_{n} \leqslant H_{t} / L_{n}$ being an absolute upper limit when $H_{a} \ll H$.

The equations appropriate for this case differ little in form from those used to derive the results of the previous section. The term multiplied by $\Lambda$ may be omitted in the momentum Eq. (38) as already noted and so can $\rho_{d 0} / \rho_{a 0}$. With these simplifications the momentum Eq. (38) becomes

$$
\frac{d M^{*}}{d z^{*}}=\frac{m_{d}^{*}}{\rho_{d}^{*}\left(w^{*}+V_{N}\right)}+\frac{F_{s}^{*}}{w^{*}} .
$$

Here and in the mass dissolution Eq. (40) the density $\rho_{d}^{*}$ is now a function of $z^{*}$ according to Eq. (81). There are other more subtle differences because the correlations used for the Sherwood number and the bubble rise velocity all depend on the bubble diameter which changes not only due to dissolution as before but, in this case, also due to expansion. 

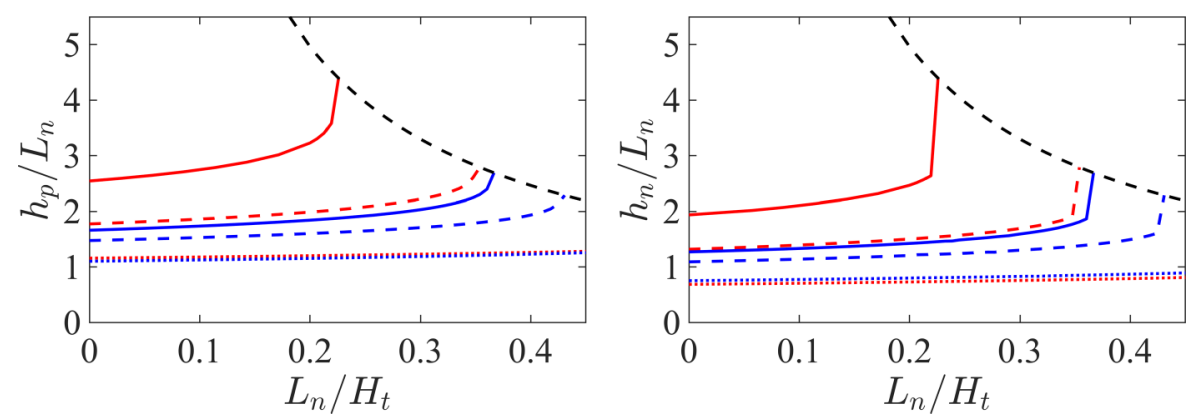

FIG. 10. Peel height (left) and neutral height in shallow water as a function of the parameter $L_{n} / H_{t}$ expressing the ratio of the estimated neutral height to the total hydrostatic head at the plume source; water depth increases from right to left. The lines have been calculated with $p_{\text {atm }} /\left(\rho_{a} g\right)=H_{a}=10 \mathrm{~m}$. The dashed black lines denote the upper limit when the peel and the neutral heights $h_{p}$ and $h_{n}$ equal the water depth $H$; in the latter case, there is no peeling. The solid lines are for $\Theta=0$, the dashed lines for $\Theta=1$, and the dotted lines for $\Theta=4$; red lines are for $V_{N}=0$ and blue lines for $V_{N}=2$.

Figure 10 shows the dependence of $h_{p}$ and $h_{n}$ on the parameter $L_{n} / H_{t}$; the dashed black line is the upper limit $h_{p}=H$. The solid lines are for $\Theta=0$, the dashed lines for $\Theta=1$ and the dotted lines for $\Theta=4$; red lines are for $V_{N}=0$ and blue lines for $V_{N}=2$. According to Eq. (75), a bubble with a diameter of $60 \mathrm{~mm}$ has a rise velocity of $w_{b}=0.55 \mathrm{~m} / \mathrm{s}$. Thus, $V_{N}=2$ in this case would correspond to $L_{n} N=0.275 \mathrm{~m} / \mathrm{s}$.

Nondissolving bubbles produce higher peel and neutral heights, and smaller rise velocities also increase the peel height as found in the deep-water case. As the dissolution rate increases, bubbles dissolve more and more quickly before they have a chance to feel the decreasing hydrostatic head and the sensitivity of the peel height to the water depth correspondingly decreases. An interesting remark that can be made in connection with Fig. 10 is that the largest values of the peel and neutral heights can be $50 \%$ larger than in deep water due to the increased buoyancy provided by the expanding bubbles.

The rate of growth of the peel and neutral heights with decreasing water depth sharply increases as it gets close to the water depth. The reason is that a small fractional decrease of the hydrostatic pressure requires a proportional increase of the bubble volume. If the bubbles have grown significantly rising from the source, then this fractional volume increase corresponds to a large volume increase in absolute terms and a corresponding increase in buoyancy.

\section{SUMMARY AND CONCLUSIONS}

This paper has presented a scaling analysis of bubble and drop plumes in a stratified ambient including the effects of mass transfer between the bubbles/drops and the ambient liquid. The work is based on a standard top-hat integral model, which can be readily reduced to a Gaussian model as explained in our earlier paper Ref. [7]. To make the investigation of parameter space manageable we have focused on the limit case of a point source, to which more realistic source conditions can be reduced by appealing to the notion of virtual source (see, e.g., Ref. [65]).

The results for the peel and neutral heights have been found to be primarily dependent on two dimensionless parameters: $\Theta$, which compares the bubble dissolution rate with the rise time to the neutral height, and $\Lambda$, which accounts for the effect of the dissolved material on the liquid density and, therefore, buoyancy. For bubbles, this latter parameter is very small in shallow water, but becomes more important in deep water where the hydrostatic pressure increases the ratio between the gas and liquid densities. For drops, $\Lambda$ is typically of order 1 and can be positive when the dissolved material makes the liquid lighter, as in the case of methane and water, or negative, when the dissolved material makes the liquid heavier, as in the case of $\mathrm{CO}_{2}$ and water. In the former case 
the buoyancy lost upon dissolution of the drops/bubbles can be restored, fully or in part, by the modification of the liquid density. We have shown that the neglect of this effect, as in much of the literature, may be unjustified in some situations.

A third important parameter is the drop/bubble rise velocity compared with the characteristic plume velocity. A small value of this parameter corresponds to a strong coupling of the disperse phase with the liquid and results in an increase of the neutral and peel heights.

Drop plumes in deep or shallow water behave similarly in view of the approximate incompressibility of liquids. For bubbles, however, the situation is very different. When the peel height is small compared to the total water depth, expansion due to the falling hydrostatic pressure is a minor effect. When peel height and water depth are comparable, however, bubble expansion can play a significant role if the dissolution rate is slow enough that bubbles can rise close to the free surface. In this case, the peel and neutral heights can be significantly larger than in deep water.

An important process that has not been considered is the fall-back of the liquid from the peel height to the intrusion height. This extension would require some version of the so-called doubleplume model $[6,44]$ and will be pursued in a future paper.

\section{ACKNOWLEDGMENTS}

This research was made possible by a grant from the Gulf of Mexico Research Initiative. Data are publicly available through the Gulf of Mexico Research Initiative Information \& Data Cooperative (GRIIDC) at https://data.gulfresearchinitiative.org doi:10.7266/N7H9939V.

\section{APPENDIX}

In the development of the mathematical formulation of the model and in its simplification we have made several assumptions that we now justify quantitatively.

\section{Neglect of $\alpha_{d}$}

In Sec. II we have neglected the disperse-phase volume fraction everywhere except in $m_{d}$, the flow rate of the disperse phase. We now justify this procedure ignoring the fact that the area occupied by the bubble is smaller than that of the plume liquid since the conclusion of the argument is that $\alpha_{d}$ is negligible.

We consider the ratio between the volumetric flow rates of the disperse phase, $m_{d} / \rho_{d}$ and of the plume, $m / \rho$ :

$$
\frac{m_{d} / \rho_{d}}{m / \rho}=\frac{\alpha_{d}}{1-\alpha_{d}}\left(1+\frac{w_{d}}{w}\right)
$$

or, upon using the relation between $F_{d}$ and $m_{d}$ given in Eq. (21), the definition Eq. (33) of the characteristic neutral height $L_{n}$ and of the dimensionless mass flow rate Eq. (34),

$$
\frac{\alpha_{d}}{1-\alpha_{d}}\left(1+\frac{w_{d}}{w}\right)=\frac{\rho}{\rho-\rho_{d}} \frac{L_{n} N^{2}}{g} \frac{F_{d}}{F_{d 0}} \frac{1}{m^{*}}=\frac{\rho}{\rho-\rho_{d}}\left[-\frac{d}{d z^{*}}\left(\frac{\rho}{\rho_{a 0}}\right)\right] \frac{F_{d}}{F_{d 0}} \frac{1}{m^{*}} .
$$

Since, as is readily shown,

$$
\frac{L_{n} N^{2}}{g}=\frac{d}{d z^{*}} \frac{\rho}{\rho_{a 0}}
$$

this term therefore represents the relative change of the ambient water density over a distance of the order of the neutral height and is typically of the order of a few parts in $10^{2}-10^{3}$. For any problem in which the disperse-phase buoyancy is significant, the first fraction in Eq. (A2) will be a number greater than 1, but not by an order of magnitude. For example, for oil drops, it would be about 5 , and much closer to 1 for gas bubbles. Since the disperse phase dissolves, $F_{d} / F_{d 0} \leqslant 1$. The 
only large contribution to Eq. (A2) comes from $m^{*}$ which, for the limit case of a pointlike source, vanishes at the source and, due to the nondimensionalization explained in Sec. III, is a number of order one at the neutral height. By considering the limit case $\Lambda=1$ and $V_{N} \ll w^{*}$, it is easy to show from Eqs. (37), (39), and (57) that, near the source, $m^{*} \propto\left(z^{*}\right)^{5 / 3}$. Thus, $m^{*} \sim\left|\left(d / d z^{*}\right)\left(\rho / \rho_{a 0}\right)\right|$ for $z^{*} \sim\left|\left(d / d z^{*}\right)\left(\rho / \rho_{a 0}\right)\right|^{3 / 5}$, which equals about 0.06 and 0.02 for $\left|\left(d / d z^{*}\right)\left(\rho / \rho_{a 0}\right)\right|^{3 / 4}=10^{-2}$ and $10^{-3}$, respectively. We therefore conclude that, except for a very small fraction of the plume height, the assumption $\alpha_{d} \ll 1$ is very well justified.

\section{Equation (40)}

Avoiding the simplification stemming from $\rho_{d \text {,dslv }} \ll \rho_{\text {sat }}$, the dimensionless form of Eq. (40) for $m_{d}^{*}$ is

$$
\frac{d m_{d}^{*}}{d z^{*}}=\Theta \frac{\mathrm{Sh}^{*}}{w^{*}+V_{N}}\left(\frac{m_{d}^{*}}{\rho_{d}^{*}}\right)^{1 / 3}\left(\frac{\rho_{d, \mathrm{dslv}}}{\rho_{\mathrm{sat} 0}}-\rho_{\mathrm{sat}}^{*}\right) .
$$

Since the solubility tends to decrease with pressure, $\rho_{d \text {,dslv }} \leqslant \rho_{\text {sat } 0}$, and the first term in parentheses is certainly less than, or equal to, 1 . If $n$ drops/bubbles per unit volume, each one containing a mass $m_{1}$ of soluble material, dissolve completely, then the resulting density of dissolved material in the ambient liquid is $n m_{1}=\alpha_{d} \rho_{\text {sat }}$. We thus conclude that $\rho_{d \text {,dslv }} / \rho_{\text {sat } 0} \leqslant \alpha_{d}$ and, therefore, small over most of the plume as concluded before.

\section{Multicomponent drop}

The model of Sec. IV, in which drops are assumed to consist of a single soluble component, can be readily extended to a multicomponent drop only some constituents of which are soluble as follows.

We express the density of such a drop as

$$
\rho_{\text {drop }}=\left(1-\alpha_{B}\right) \rho_{A}+\alpha_{B} \rho_{B}
$$

where $\alpha_{B}$ is the volume fraction of the soluble components, and $\rho_{A}, \rho_{B}$ are the (mean) densities of the insoluble and soluble constituents, respectively. In principle, each soluble component will have different solubility. In what follows we neglect the solubility difference for simplicity, although the model can be readily extended to include this effect.

The mass flux of the insoluble component in the plume is

$$
m_{A}=\left(1-\alpha_{B}\right) \rho_{A} A_{\mathrm{drop}}\left(w+w_{\mathrm{drop}}\right)
$$

The mass fluxes of undissolved and dissolved $B$ components are

$$
\begin{gathered}
m_{B}=\alpha_{B} \rho_{B} A_{\mathrm{drop}}\left(w+w_{\mathrm{drop}}\right), \\
m_{B, \mathrm{dslv}}=\rho_{B, \mathrm{dslv}} A_{l} w .
\end{gathered}
$$

The conservation relations for $A, B$ are $m_{A}=m_{A 0}, m_{B}+m_{B, \mathrm{dslv}}=m_{B 0}$.

The momentum equation for the multicomponent case is, then,

$$
\frac{d M}{d z}=\frac{\rho_{a}-\rho_{A}}{\rho_{A}} \frac{m_{A} g}{w+w_{d}}+\frac{\rho_{a}-\rho_{B}}{\rho_{B}} \frac{m_{B} g}{w+w_{d}}-\frac{\beta_{B} m_{B, \mathrm{dsl} v} g}{w}+\frac{F_{S}}{w} .
$$

Given the similarity of this equation with the momentum Eq. (24) used in the analysis of the main text, it is clear how to apply scaling arguments parallel to those of Sec. III to find two new dimensionless parameters. 
[1] G. R. Hunt and T. S. van den Bremer, Classical plume theory: 1937-2010 and beyond, IMA J. Appl. Math. 76, 424 (2011).

[2] A. W. Woods, Turbulent plumes in nature, Annu. Rev. Fluid Mech. 42, 391 (2010).

[3] M. K. McNutt, R. Camilli, T. J. Crone, G. D. Guthrie, P. A. Hsieh, T. B. Ryerson, O. Savas, and F. Shaffer, Review of flow rate estimates of the Deepwater Horizon oil spill, Proc. Natl. Acad. Sci. USA 109, 20260 (2012).

[4] C. M. Reddy, J. S. Arey, J. S. Seewald, S. P. Sylva, K. L. Lemkau, R. K. Nelson, C. A. Carmichael, C. P. McIntyre, J. Fenwick, G. T. Ventura, B. A. S. van Mooy, and R. Camilli, Composition and fate of gas and oil released to the water column during the Deepwater Horizon oil spill, Proc. Natl. Acad. Sci. USA 109, 20229 (2012).

[5] T. B. Ryerson, R. Camilli, J. D. Kessler, E. B. Kujawinski, C. M. Reddy, D. L. Valentine, E. Atlas, D. R. Blake, J. de Gouw, S. Meinardi, D. D. Parrish, J. Peischl, J. S. Seewald, and C. Warneke, Chemical data quantify Deepwater Horizon hydrocarbon flow rate and environmental distribution, Proc. Natl. Acad. Sci. USA 109, 20246 (2012).

[6] T. J. McDougall, Bubble plumes in stratified environments, J. Fluid Mech. 85, 655 (1978).

[7] S. Chu and A. Prosperetti, Bubble plumes in a stratified environment: Source parameters, scaling, intrusion height, and neutral height, Phys. Rev. Fluids 2, 104503 (2017).

[8] L. Zheng and P. D. Yapa, Modeling gas dissolution in deepwater oil/gas spills, J. Marine Syst. 31, 299 (2002).

[9] M. G. Domingos and S. S. S. Cardoso, Turbulent two-phase plumes with bubble-size reduction owing to dissolution or chemical reaction, J. Fluid Mech. 716, 120 (2013).

[10] F. A. Bombardelli, G. C. Buscaglia, C. R. Rehmann, L. E. Rincón, and M. H. García, Modeling and scaling of aeration bubble plumes: A two-phase flow analysis, J. Hydraul. Res. ASCE 45, 617 (2007).

[11] G. C. Buscaglia, F. A. Bombardelli, and M. H. Garcia, Numerical modeling of large-scale bubble plumes accounting for mass transfer effects, Int. J. Multiphase Flow 28, 1763 (2002).

[12] J. H. Milgram, Mean flow in round bubble plumes, J. Fluid Mech. 133, 345 (1983).

[13] A. Wüest, N. H. Brooks, and D. M. Imboden, Bubble plume modeling for lake restoration, Water Res. Res. 28, 3235 (1992).

[14] A. Fabregat, B. Deremble, N. Wienders, A. Stroman, A. Poje, T. M. Özgökmen, and W. K. Dewar, Rotating 2D point source plume models with application to Deepwater Horizon, Ocean Model. 119, 118 (2017).

[15] A. Fabregat, W. K. Dewar, T. M. Özgökmen, A. C. Poje, and N. Wienders, Numerical simulations of turbulent thermal, bubble, and hybrid plumes, Ocean Model. 90, 16 (2015).

[16] P. van Reeuwijk, P. Salizzoni, G. R. Hunt, and J. Craske, Turbulent transport and entrainment in jets and plumes: A DNS study, Phys. Rev. Fluids 1, 074301 (2016).

[17] D. Yang, B. Chen, S. A. Socolofsky, M. Chamecki, and C. Meneveau, Large-eddy simulation and parametrization of buoyant plume dynamics in stratified flow, J. Fluid Mech. 794, 798 (2016).

[18] A. Fabregat Tomàs, A. C. Poje, T. M. Özgökmen, and W. K. Dewar, Dynamics of multiphase turbulent plumes with hybrid buoyancy sources in stratified environments, Phys. Fluids 28, 095109 (2016).

[19] B. C. Crounse, E. J. Wannamaker, and E. E. Adams, Integral model of a multiphase plume in quiescent stratification, J. Hydraul. Eng. ASCE 133, 70 (2007).

[20] C. Spier, W. T. Stringfellow, T. C. Hazen, and M. Conrad, Distribution of hydrocarbons released during the 2010 MC252 oil spill in deep offshore waters, Environ. Pollut. 173, 224 (2013).

[21] H. E. Huppert and J. S. Turner, Double-diffusive convection, J. Fluid Mech. 106, 299 (1981).

[22] T. Radko, Double-diffusive Convection (Cambridge University Press, Cambridge, 2013).

[23] R. W. Schmitt, Double diffusion in oceanography, Annu. Rev. Fluid Mech. 26, 255 (1994).

[24] S. A. Socolofsky and E. E. Adams, Role of slip velocity in the behavior of stratified multiphase plumes, J. Hydraul. Eng. ASCE 131, 273 (2005).

[25] C. A. Koh, E. D. Sloan, A. K. Sum, and D. T. Wu, Fundamentals and applications of gas hydrates, Annu. Rev. Chem. Biomol. Eng. 2, 237 (2011).

[26] P. D. Yapa, M. R. Wimalaratne, A. L. Dissanayake, and J. A. DeGraff, How does oil and gas behave when released in deepwater? J. Hydro-Environ. Res. 6, 275 (2012). 
[27] R. P. Warzinski, R. Lynn, I. Haljasmaa, I. Leifer, F. Shaffer, B. J. Anderson, and J. S. Levine, Dynamic morphology of gas hydrate on a methane bubble in water: Observations and new insights for hydrate film models, Geophys. Res. Lett. 41, 6841 (2014).

[28] T. Asaeda and J. Imberger, Structure of bubble plumes in linearly stratified environments, J. Fluid Mech. 249, 35 (1993).

[29] C. J. Lemckert and J. Imberger, Energetic bubble plumes in arbitrary stratification, J. Hydraul. Eng. ASCE 119, 680 (1993).

[30] R. C. Clift, J. R. Grace, and M. E. Weber, Bubbles, Drops, and Particles (Academic Press, San Diego, CA, 1978).

[31] J. C. Scott, The preparation of water for surface-clean fluid mechanics, J. Fluid Mech. 69, 339 (1975).

[32] Y. H. Tsang, Y. H. Koh, and D. L. Koch, Bubble-size dependence of the critical electrolyte concentration for inhibition of coalescence, J. Colloid Interface Sci. 275, 290 (2004).

[33] U. C. Bandara and P. D. Yapa, Bubble sizes, breakup, and coalescence in deepwater gas/oil plumes, J. Hydraul. Eng. 137, 729 (2011).

[34] L. Zhao, M. C. Boufadel, K. Lee, T. King, N. Loney, and X. Geng, Evolution of bubble size distribution from gas blowout in shallow water, J. Geophys. Res. Oceans 121, 1573 (2016).

[35] P. A. Rona, K. G. Bemis, C. D. Jones, D. R. Jackson, K. Mitsuzawa, and D. Silver, Entrainment and bending in a major hydrothermal plume, Main Endeavour Field, Juan de Fuca Ridge, Geophys. Res. Lett. 33, L19313 (2006).

[36] S. Chu and A. Prosperetti, Dissolution and growth of a multicomponent drop in an immiscible liquid, J. Fluid Mech. 798, 787 (2016).

[37] B. R. Morton and J. Middleton, Scale diagrams for forced plumes, J. Fluid Mech. 58, 165 (1973).

[38] G. R. Hunt and N. B. Kaye, Lazy plumes, J. Fluid Mech. 533, 329 (2005).

[39] N. B. Kaye, Turbulent plumes in stratified environments: A review of recent work, Atmos. Ocean 46, 433 (2008).

[40] B. R. Morton, G. Taylor, and J. S. Turner, Turbulent gravitational convection from maintained and instantaneous sources, Proc. R. Soc. London A 234, 1 (1956).

[41] J. S. Turner, Turbulent entrainment: the development of the entrainment assumption and its application to geophysical flows, J. Fluid Mech. 173, 431 (1986).

[42] G. Carazzo, E. Kamiski, and S. Tait, The route to self-similarity in turbulent jets and plumes, J. Fluid Mech. 547, 137 (2006).

[43] A. C. H. Lai, S. N. Chan, A. W. K. Law, and E. E. Adams, Spreading hypothesis of a particle plume, J. Hydraul. Eng. 142, 04016065 (2016).

[44] S. A. Socolofsky, T. Bhaumik, and D. G. Seol, Double-plume integral models for near-field mixing in multiphase plumes, J. Hydraul. Eng. ASCE 134, 772 (2008).

[45] A. W. Woods and C. P. Caulfield, A laboratory study of explosive volcanic eruptions, J. Geophys. Res. 97, 6699 (1992).

[46] J. S. Turner, Buoyancy Effects in Fluids (Cambridge University Press, Cambridge, 1979).

[47] S. A. Socolofsky, E. E. Adams, and C. R. Sherwood, Formation dynamics of subsurface hydrocarbon intrusions following the Deepwater Horizon blowout, Geophys. Res. Lett. 38, L09602 (2011).

[48] E. W. Lemmon, M. O. McLinden, and D. G. Friend, Thermophysical properties of fluid systems, in NIST Chemistry WebBook, NIST Standard Reference Database Number 69, edited by P. J. Linstrom and W. G. Mallard (National Institute of Standards and Technology, Gaithersburg, MD, 2018).

[49] J. C. Moore, R. Battino, T. R. Rettich, Y. P. Handa, and E. Wilhelm, Partial molar volumes of "gases" at infinite dilution in water at 298.15 K, J. Chem. Eng. Data 27, 22 (1982).

[50] P. Hyncica, L. Hnedkovksy, and I. Cibulka, Partial molar volumes of organic solutes in water. X. Benzene and toluene at temperatures from (298 to 573 ) $\mathrm{K}$ and at pressures up to $30 \mathrm{MPa}$, J. Chem. Thermodyn. 35, 1905 (2003).

[51] R. J. Lewis, Xylene, in Hawley's Condensed Chemical Dictionary, 14th ed. (Wiley and Sons, New York, 2001), pp. 1183-1185.

[52] S. H. Yalkowsky and Y. He, Handbook of Aqueous Solubility Data (CRC Press, New York, 1992). 
[53] S. Sawamura, K. Suzuki, and Y. Taniguchi, Effect of pressure on the solubilities of o-, m-, and p-xylene in water, J. Solution Chem. 16, 649 (1987).

[54] N. Spycher, K. Pruess, and J. Ennis-King, $\mathrm{CO}_{2}-\mathrm{H}_{2} \mathrm{O}$ mixtures in the geological sequestration of $\mathrm{CO}_{2}$. I. Assessment and calculation of mutual solubilities from 12 to $100^{\circ} \mathrm{C}$ and up to 600 bar, Geochim. Cosmochim. Acta 67, 3015 (2003).

[55] S. Budavari, M. J. O’Neil, A. Smith, and P. E. Heckelman, Benzene, in The Merck Index: An Encyclopedia of Chemicals, Drugs, and Biologicals (Merck, and Co., Rahway, NJ, 2001), pp. 182-183.

[56] National Library of Medicine, Hazardous substances data bank, Tech. Rep. National Institutes of Health, http://toxnet.nlm.nih.gov (2010).

[57] L. D. Landau and E. M. Lifshitz, Statistical Physics, 3rd ed. (Elsevier, Amsterdam, 1969).

[58] J. M. Prausnitz, R. N. Lichtenthaler, and E. Gomes de Azevedo, Molecular Thermodynamics of FluidPhase Equilibria, 3rd ed. (Prentice-Hall, Englewood Cliffs, NJ, 1999).

[59] R. Sander, Compilation of Henry's law constants (version 4.0) for water as solvent, Atmos. Chem. Phys. 15, 4399 (2015).

[60] D. Rodrigue, A general correlation for the rise velocity of single gas bubbles, Can. J. Chem. Engng. 82, 382 (2004).

[61] V. G. Levich, Physicochemical Hydrodynamics (Prentice-Hall, Englewood Cliffs, NJ, 1962).

[62] G. A. Hughmark, Liquid-liquid spray column drop size, holdup, and continuous phase mass transfer, Ind. Eng. Chem. Fundam. 58, 107 (1967).

[63] D. Legendre, R. Zenit, and J. R. Velez-Cordero, On the deformation of gas bubbles in liquids, Phys. Fluids 24, 043303 (2012).

[64] E. Michaelides, Particles, Bubbles, and Drops: Their Motion, Heat and Mass Transfer (World Scientific, Singapore, 2006).

[65] B. R. Morton, Forced plumes, J. Fluid Mech. 5, 151 (1959). 